Reconstruction algorithm for the linearized polarization tomography problem with incomplete data.

Lionheart, William and Sharafutdinov, Vladimir

2008

MIMS EPrint: 2008.75

Manchester Institute for Mathematical Sciences

School of Mathematics

The University of Manchester

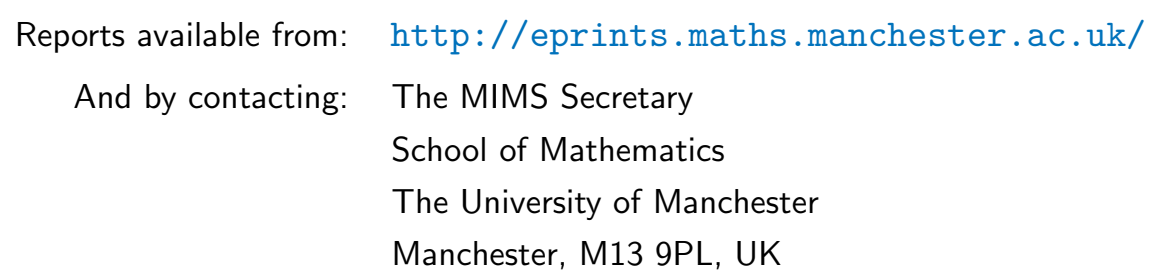

ISSN 1749-9097 


\title{
Reconstruction algorithm for the linearized polarization tomography problem with incomplete data.
}

\author{
William Lionheart and Vladimir Sharafutdinov
}

\begin{abstract}
The paper studies some problem of polarization tomography by the method developed in [12]. Specifically it is shown that the Truncated Transverse Ray Transform (TTRT) restricted to lines orthogonal to one of three generically chosen directions determines a trace-free symmetric tensor field uniquely. While a constructive formula is given for the solution this is unstable. However for six suitably chosen directions a stable reconstruction algorithm is given. The result has applications to photoelastic tomography.
\end{abstract}

\section{Introduction}

Many physical quantities such as stress and permittivity can be represented by symmetric second rank tensor fields and this gives rise to a need to determine such quantities in the interior of an object using only external measurements. Where the tensor is not a scalar multiple of the Kronecker tensor such problems are anisotropic. In contrast to the isotropic case, the subject of anisotropic inverse problems is not well developed with few results showing the sufficiency of data to determine the unknown tensor field uniquely, a lack of explicit inverse procedures to solve the inverse problem and few experimental groups working on systems to collect the necessary data.

The photoelastic effect relates the stress $\sigma$ to the dielectric permittivity tensor $\varepsilon$ via the equation

$$
\varepsilon=\varepsilon_{0}+C_{0} \sigma+C_{1} \operatorname{tr}(\sigma) \delta
$$

where $C_{0}$ and $C_{1}$ are constants depending on the material, $\varepsilon_{0}$ is the permittivity tensor in the unstressed state (typically isotropic) and $\delta$ is the Kronecker tensor. The resulting anisotropic permittivity tensor effects the propagation of light in a transparent medium. In the case of $\epsilon_{0}=$ const. $\delta$ and of a small stress, and consequently a permittivity close to isotropic, light will continue to propagate in a straight line through material but the polarization state of the light will change $[\mathbf{1 1}, \mathrm{Ch}$ 5]. For sufficiently small (in the $C^{1}$ sense) $\sigma$ the dependence of the change in the polarization state of the light on $\varepsilon$ for each ray path is approximated by a linear operator, the truncated transverse ray transform TTRT, which is the subject of this paper. In many practical experiments the data that can be measured are exactly the characteristic parameters $[\mathbf{1}],[\mathbf{5}]$. In this linear case these give exactly two independent measurements per ray. Typically the overall phase change of the light as it

1991 Mathematics Subject Classification. Primary 44A12, 45Q05; Secondary 53C65, 78A05.

Key words and phrases. Tensor tomography, photoelasicity, polarized light.

Partially supported by EPSRC grants GR/R86300/01, EP/E010997/1, EP/F013329/1, EP/C509803/1, and EP/F033974/1.

Partially supported by CRDF grant KAM1-2851-AL-07 and RFBR grant 08-01-92001-NNS. 
passes through the object cannot be accurately measured, but if it were the transverse ray transform TRT rather than the TTRT would be the appropriate operator.

It is often helpful to use the natural orthogonal splitting of symmetric rank two tensors in to the kernel of the trace operator and its orthogonal complement, which consists of scalar multiples of the Kronecker tensor. In elasticity the trace-free part of a tensor $f-(1 / 3) \operatorname{tr}(f) \delta$ for $f$ a symmetric second rank tensor field on $\mathbb{R}^{3}$, is called the deviatoric part. It is shown in [11, Thm 6.2.2] that the null space of the TTRT is exactly the scalar multiples of the Kronecker tensor.

In photoelastic tomography one attempts to recover the deviatoric part of the permittivity $\varepsilon$, and hence the deviatoric part of the stress tensor, from measurements of characteristic parameters for a large number of rays. That is to invert the TTRT from some subset of data. The method has been used successfully by Aben et al [3] to image one component of the stress tensor of an object from measurements of polarized light transmitted through the object while the object is rotated $180^{\circ}$ about an axis. The main interest of Aben's group is in the measurement of residual stress in glass and they make the reasonable assumption that the stress tensor $\sigma$ (a symmetric second rank tensor field) is solenoidal, that is

$$
\sum_{j=1}^{3} \frac{\partial \sigma_{i j}}{\partial x_{j}}=0 .
$$

In the case of glass objects one can assume that the object $D$, that is the support of $\sigma$, is bounded by a smooth surface, or at worst has only corners and edges (for example Lipschitz). If we interpret (1.2) in the distributional sense this is equivalent, where the support has a smooth boundary, with the assumption that the normal component vanishes at the boundary $\sigma N=0$ on $\partial D$ where $N$ is the unit normal. Under these assumptions a reconstruction algorithm was proposed by the second author [10], [11, Sec 2.16] and tested on simulated data, and a practical realization of a simple related algorithm was implemented by Aben et al [3] and applied to experimental data. These algorithms rely on data derived from rotation about one fixed axis defined by the unit vector $\eta$, that is data from rays orthogonal to $\eta$, and recover the $\eta \cdot \sigma \eta$ component of the stress. In each plane normal to $\eta$ intersecting the support of $\sigma$ these algorithms use filtered back projection methods common in twodimensional x-ray tomography. The algorithms essentially work "slice-by-slice". They can therefore be implemented easily and efficiently. By rotation of the object about five generically chosen axes one can recover all components of the deviatoric part of $\varepsilon$ and hence knowing $C_{0}$ the deviatoric part of $\sigma$. The solenoidal condition (1.2), together with the vanishing of $\sigma$ at infinity, then means that $\sigma$ itself is uniquely determined.

There are however situations in which photoelastic tomography may prove useful where the solenoidal condition is violated, either by the presence of body forces in the interior of the object or more importantly because external loads are applied to the object. An important example is where a transparent object is made of a mechanical component and an external load applied. It is possible to freeze the stress in to the object and then examine the object with polarized light. The traditional procedure for three dimensional stress analysis [1, p.63], [4] is to carefully cut the model in to thin planar slices. The optical response of each slice is measured using a polarimeter and this used to estimate the difference between the principle stresses in the slice. It has been proposed [15] that a tomographic method is used so as to avoid the lengthy and expensive process of cutting sections, and to avoid the destruction of the test object. However to date the only known analytical algorithm [11, Sec 6.6] for the inversion of the TTRT assumes data is measured from all ray directions. This would be difficult to arrange in practice and the process of acquiring the data would be time consuming. A numerical study [14] using a discretization to reduce the problem to a sparse system of linear equations, indicated that a stable reconstruction with a finite number of axes might be possible.

Other possible applications of tomography with polarized light include the Kerr effect [2], tomography of plasma $[\mathbf{9}]$, and weakly birefringent liquid crystals $[\mathbf{6}]$. 
In this paper we demonstrate that there is indeed a reconstruction algorithm for the TTRT for a general tensor field using data from only three rotation axes (or normal directions) and a stable reconstruction algorithm for six axes. The method first performs a slice by slice reconstruction of six functions by two dimensional back projection and filter methods. The components of tensor field are related to these functions by a linear operator with coefficients that are rational functions of the Fourier transform variables.

If the reader is not familiar with the truncated transverse ray transform, we recommend Section 1 of the paper [13] for the physical motivation of TTRT. The latter paper studies the nonlinear inverse problem of polarization tomography for a nonhomogeneous background medium. While uniqueness of solution is established for the non-linear problem for a symmetric tensor field, uniqueness does not hold for the non-symmetric case $[\mathbf{8}]$. In contrast to $[\mathbf{1 3}]$ and $[\mathbf{8}]$, the present paper is restricted to studying the linearized inverse problem in the case of a homogeneous background medium, but only incomplete data are assumed to be known.

\section{Posing the problem and discussion of results}

We start with discussing tensor fields on $\mathbb{R}^{n}$ for arbitrary $n$. But only the cases $n=2$ and $n=3$ are used in the main part of the article.

We consider $\mathbb{R}^{n}$ as the Euclidean vector space with the standard scalar product $\langle\cdot, \cdot\rangle$ and norm $|\cdot|$. Since we are going to use the Fourier transform, $\mathbb{R}^{n}$ is considered as the real part of $\mathbb{C}^{n}$; the scalar product is extended to the Hermitian product on $\mathbb{C}^{n}$ which is again denoted by $\langle\cdot, \cdot\rangle$.

Let $S^{2} \mathbb{C}^{n}$ be the complex vector space of symmetric $\mathbb{R}$-bilinear maps $\mathbb{R}^{n} \times \mathbb{R}^{n} \rightarrow \mathbb{C}$. Elements of the space are (complex-valued) symmetric tensors of second rank on $\mathbb{R}^{n}$. Any such tensor $f \in S^{2} \mathbb{C}^{n}$ is identified with the $\mathbb{C}$-linear operator $f: \mathbb{C}^{n} \rightarrow \mathbb{C}^{n}$ by the formula $\langle f \xi, \eta\rangle=\langle f \eta, \xi\rangle=f(\xi, \eta)$ for $\xi, \eta \in \mathbb{R}^{n}$. If $f^{*}$ is the dual operator, then $f^{*}=\bar{f}$. Operators satisfying the latter equation will be called symmetric operators. By $\delta$ we denote the Kronecker tensor that corresponds to the identity operator.

Given an orthonormal basis $\left(e_{1}, \ldots, e_{n}\right)$ of $\mathbb{R}^{n}$, a tensor $f \in S^{2} \mathbb{C}^{n}$ can be represented by the symmetric $n \times n$-matrix $\left(f_{j k}\right), f_{j k}=f\left(e_{j}, e_{k}\right)$. The Hermitian scalar product on $S^{2} \mathbb{C}^{n}$ is defined by $\langle f, g\rangle=\sum_{j, k=1}^{n} f_{j k} \bar{g}_{j k}$, the result is independent of the choice of an orthonormal basis. We use only orthonormal bases, so we do not distinguish co- and contravariant tensors.

For a unit vector $\xi \in \mathbb{R}^{n}$, let $Q_{\xi}: S^{2} \mathbb{C}^{n} \rightarrow S^{2} \mathbb{C}^{n}$ be the orthogonal projection onto the subspace $\left\{f \in S^{2} \mathbb{C}^{n} \mid f \xi=0, \operatorname{tr} f=\sum_{j=1}^{n} f_{j j}=0\right\}$. For example for $n=3$, for an orthonormal basis of the form $\left(e_{1}, e_{2}, e_{3}=\xi\right)$, the projection is expressed by

$$
Q_{\xi} f=\frac{1}{2}\left(\begin{array}{ccc}
f_{11}-f_{22} & 2 f_{12} & 0 \\
2 f_{21} & f_{22}-f_{11} & 0 \\
0 & 0 & 0
\end{array}\right) .
$$

Let us recall the definition of the Schwartz space $\mathcal{S}\left(\mathbb{R}^{n}\right)$ of smooth rapidly decaying functions. The space consists of (complex-valued) functions $f \in C^{\infty}\left(\mathbb{R}^{n}\right)$ satisfying $\left|\partial^{\alpha} f(x)\right| \leq C_{\alpha, k}(1+|x|)^{-k}$ for every multi-index $\alpha$ and every integer $k \geq 0$. The Fourier transform $F: \mathcal{S}\left(\mathbb{R}^{n}\right) \rightarrow \mathcal{S}\left(\mathbb{R}^{n}\right)$ is defined by

$$
F[f]=\hat{f}(y)=(2 \pi)^{-n / 2} \int_{\mathbb{R}^{n}} e^{-i\langle y, x\rangle} f(x) d x .
$$

We will need also the partial Fourier transform $F_{V}: \mathcal{S}\left(\mathbb{R}^{n}\right) \rightarrow \mathcal{S}\left(\mathbb{R}^{n}\right)$ that is defined for any vector subspace $V \subset \mathbb{R}^{n}$ as follows. Choose Cartesian coordinates $\left(x_{1}, \ldots, x_{n}\right)$ in $\mathbb{R}^{n}$ such that $V=\left\{x \mid x_{k+1}=\cdots=x_{n}=0\right\}$. Then

$$
F_{V}[f]=\hat{f}\left(y_{1}, \ldots, y_{k}, x_{k+1}, \ldots, x_{n}\right)=(2 \pi)^{-k / 2} \int_{\mathbb{R}^{k}} e^{-i\left(y_{1} x_{1}+\cdots+y_{k} x_{k}\right)} f(x) d x_{1} \ldots d x_{k} .
$$

The result is independent of the choice of such coordinates and satisfies $F=F_{V} F_{V^{\perp}}=F_{V^{\perp}} F_{V}$. 
Let $\mathcal{S}\left(\mathbb{R}^{n} ; S^{2} \mathbb{C}^{n}\right)$ be the Schwartz space of $S^{2} \mathbb{C}^{n}$-valued functions on $\mathbb{R}^{n}$. Elements of the space are smooth rapidly decaying second rank symmetric tensor fields on $\mathbb{R}^{n}$. In an orthonormal basis, such a field is represented by a symmetric $n \times n$-matrix $f=\left(f_{j k}(x)\right)$ with functions $f_{j k} \in$ $\mathcal{S}\left(\mathbb{R}^{n}\right)$ that are components of $f$ (with respect to the basis). The Fourier transform $\mathcal{S}\left(\mathbb{R}^{n} ; S^{2} \mathbb{C}^{n}\right) \rightarrow$ $\mathcal{S}\left(\mathbb{R}^{n} ; S^{2} \mathbb{C}^{n}\right), f \mapsto \hat{f}$ is defined component-wise, $\hat{f}=\left(\hat{f}_{j k}\right)$. The result is independent of the choice of an orthonormal basis. The Fourier transform on the Schwartz space $\mathcal{S}\left(\mathbb{R}^{n} ; \mathbb{C}^{n}\right)$ of vector fields is defined in the same way.

The family of oriented lines in $\mathbb{R}^{n}$ is parameterized by points of the manifold

$$
T \mathbb{S}^{n-1}=\left\{(\xi, x) \in \mathbb{R}^{n} \times \mathbb{R}^{n}|| \xi \mid=1,\langle\xi, x\rangle=0\right\} \subset \mathbb{R}^{n} \times \mathbb{R}^{n}
$$

that is the tangent bundle of the unit sphere $\mathbb{S}^{n-1} \subset \mathbb{R}^{n}$. Namely, an oriented line $l \subset \mathbb{R}^{n}$ is uniquely represented as $l=\{x+t \xi \mid t \in \mathbb{R}\}$ with $(\xi, x) \in T \mathbb{S}^{n-1}$. The Schwartz space $\mathcal{S}\left(T \mathbb{S}^{n-1}\right)$ consists of $^{-1}$ functions $\varphi \in C^{\infty}\left(T \mathbb{S}^{n-1}\right)$ satisfying the estimates

$$
\sup _{(\xi, x) \in T \mathbb{S}^{n-1}}(1+|x|)^{k}\left|\partial_{\xi}^{\alpha} \partial_{x}^{\beta} \varphi(\xi, x)\right| \leq C_{\alpha, \beta, k}
$$

for all multi-indices $\alpha, \beta$ and integers $k \geq 0$. To make sense of partial derivatives in the latter formula, the function $\varphi$ is assumed to be extended to a neighborhood of $T \mathbb{S}^{n-1}$ in $\mathbb{R}^{2 n}$ in such a way that $\varphi(t \xi, x)=\varphi(\xi, x)$ for $t>0$ and $\varphi(\xi, x+t \xi)=\varphi(\xi, x)$ for $t \in \mathbb{R}$. The space $\mathcal{S}\left(T \mathbb{S}^{n-1} ; S^{2} \mathbb{C}^{n}\right)$ of $S^{2} \mathbb{C}^{n}$-valued functions is defined in a similar way.

The truncated transverse ray transform is the linear operator

$$
K: \mathcal{S}\left(\mathbb{R}^{n} ; S^{2} \mathbb{C}^{n}\right) \rightarrow \mathcal{S}\left(T \mathbb{S}^{n-1} ; S^{2} \mathbb{C}^{n}\right)
$$

defined by

$$
K f(\xi, x)=\int_{-\infty}^{\infty} Q_{\xi} f(x+t \xi) d t .
$$

Since $Q_{\xi} \delta=0$, the operator $K$ vanishes on scalar multiples of the Kronecker tensor $\delta$, i.e., $K(\varphi \delta)=0$ for $\varphi \in \mathcal{S}\left(\mathbb{R}^{n}\right)$. Therefore we will study the problem of inverting the operator $K$ on the subspace of $\mathcal{S}\left(\mathbb{R}^{3} ; S^{2} \mathbb{C}^{3}\right)$ consisting of trace-free tensor fields, i.e., $f$ will be always assumed to satisfy

$$
\operatorname{tr} f=\sum_{j=1}^{3} f_{j j}=0 .
$$

A trace-free tensor field $f \in \mathcal{S}\left(\mathbb{R}^{3} ; S^{2} \mathbb{C}^{3}\right)$ can be uniquely recovered from the function $K f$ known on the whole of $T \mathbb{S}^{2}$, the reconstruction procedure is presented in Section 6.6 of [11].

As any 3D tomographic problem, the problem of recovering $f$ from $K f$ is overdetermined in dimension. Indeed, we are trying to recover functions $f_{j k}(x)$ of the three-dimensional argument $x \in \mathbb{R}^{3}$ given the function $K f$ on the four-dimensional manifold $T \mathbb{S}^{2}$ of lines. Therefore it is quite natural to pose the problem of recovering $f$ from incomplete data $\left.K f\right|_{L^{3}}$, where $L^{3}$ is some threedimensional submanifold of $T \mathbb{S}^{2}$. On the other hand, the problem is underdetermined in the number of unknown functions. Indeed, the matrix $(K f)(\xi, x)$ has two linearly independent components since it satisfies $\operatorname{tr}(K f)(\xi, x)=0$ and $(K f)(\xi, x) \xi=0$. A trace-free tensor field $f$ has five linearly independent components. We are trying to recover five latter unknowns from the data consisting of two components of $K f$.

In the present article, the submanifold $L^{3} \subset T \mathbb{S}^{2}$ is chosen as follows. For a unit vector $\eta \in \mathbb{S}^{2}$, let $L_{\eta}^{3}=\left\{(\xi, x) \in T \mathbb{S}^{2} \mid\langle\xi, \eta\rangle=0\right\}$ be the family of lines orthogonal to $\eta$. For a finite set $\left(\eta_{1}, \ldots, \eta_{N}\right)$ of unit vectors, we set

$$
L^{3}=L^{3}\left(\eta_{1}, \ldots, \eta_{N}\right)=\bigcup_{j=1}^{N} L_{\eta_{j}}^{3} .
$$


Our approach to the problem with data on manifold (2.3) is described as follows. We start with considering the data $\left.K f\right|_{L_{\eta}^{3}}$ for one vector. For a plane $P$ orthogonal to $\eta$, the restriction of the vector field $x \mapsto \eta \times f(x) \eta$ to $P$ can be considered as a 2 D vector field on $P$. Hereafter $\times$ is the vector product. The latter vector field will be called the slice of the vector field $\eta \times f \eta$ by the plane $P$. The slice of $f$ by $P$ is also defined, the latter being a symmetric second rank tensor field on $P$. We consider $2 \mathrm{D}$ slices of the vector field $\eta \times f \eta$ (of the tensor field $f$ ) by all 2-planes orthogonal to $\eta$ and apply a slice-by-slice reconstruction to these $2 \mathrm{D}$ vector (tensor) fields. This reconstruction procedure actually coincides, up to nonrelevant details, with the classical method of inverting the 2D Radon transform: application of the back projection operator followed by a power of the Laplacian. After passing to Fourier images, the result can be written as the pair of equations

$$
\Phi_{\eta}(y) \hat{f}(y)=\lambda_{\eta}(y), \quad \Psi_{\eta}(y) \hat{f}(y)=\mu_{\eta}(y),
$$

where $\lambda_{\eta}(y)$ and $\mu_{\eta}(y)$ are some scalar functions that have been effectively recovered from the data $\left.K f\right|_{L_{\eta}^{3}}$ in the slice-by-slice reconstruction, and $\Phi_{\eta}(y)\left(\Psi_{\eta}(y)\right)$ is a pure algebraic linear functional with coefficients depending linearly (quadratically) on $y$.

Of course, two scalar equations (2.4) are not enough for determining the tensor field $\hat{f}$. We use several unit vectors $\left(\eta_{1}, \ldots, \eta_{N}\right)$ to obtain the system

$$
\Phi_{\eta_{i}}(y) \hat{f}(y)=\lambda_{\eta_{i}}(y), \quad \Psi_{\eta_{i}}(y) \hat{f}(y)=\mu_{\eta_{i}}(y) \quad(1 \leq i \leq N)
$$

of linear algebraic equations.

How large must be the number $2 N$ of equations in system (2.5)? Since $\hat{f}$ has five linearly independent components, it might be expected that $N=3$ is sufficient for the unique reconstruction. Indeed, we will prove (Theorem 5.1) that system (2.5) is nondegenerate at almost every point $y \in \mathbb{R}^{3}$ for a generic family $\left(\eta_{1}, \eta_{2}, \eta_{3}\right)$ of three unit vectors. In particular, this is true for $\left(\eta_{1}, \eta_{2}, \eta_{3}\right)$ being an orthonormal basis of $\mathbb{R}^{3}$. This gives, in principle, a reconstruction procedure. But the reconstruction would possess some instability because system (2.5) is degenerate on some hypersurface. In the case of an orthonormal basis, system (2.5) is degenerate on coordinate planes. Since (2.5) for $N=3$ is a system of six equations in five unknowns, the right-hand side $\left(\lambda_{1}, \lambda_{2}, \lambda_{3}, \mu_{1}, \mu_{2}, \mu_{3}\right)$ must satisfy some linear equation, the consistency condition. We will write down the consistency condition explicitly in the case of an orthonormal basis.

For a stable reconstruction, we have to enlarge the number $2 N$ of equations in system (2.5). We will present a stable reconstruction procedure for the following choice of six unit vectors:

$$
\begin{cases}\eta_{1}=\frac{1}{\sqrt{2}}\left(e_{2}+e_{3}\right), & \eta_{2}=\frac{1}{\sqrt{2}}\left(e_{3}+e_{1}\right), \quad \eta_{3}=\frac{1}{\sqrt{2}}\left(e_{1}+e_{2}\right), \\ \eta_{4}=\frac{1}{\sqrt{2}}\left(e_{2}-e_{3}\right), & \eta_{5}=\frac{1}{\sqrt{2}}\left(e_{3}-e_{1}\right), \quad \eta_{6}=\frac{1}{\sqrt{2}}\left(e_{1}-e_{2}\right),\end{cases}
$$

where $\left(e_{1}, e_{2}, e_{3}\right)$ is an orthonormal basis of $\mathbb{R}^{3}$ (Theorem 6.1). In authors' opinion, the algorithm is quite suitable for usage in practical photoelasticity.

Most probably, $N=6$ is the minimal number of directions for the stable reconstruction, but the authors have not proved this statement. Another important question is about consistency conditions that are discussed in Section 7.

\section{Three kinds of ray transform}

We will need vector and tensor fields on affine subspaces (planes) of $\mathbb{R}^{n}$. For a vector subspace $V \subset \mathbb{R}^{n}$, let $V_{\mathbb{C}}$ be the complexification of $V$ and $S^{2} V_{\mathbb{C}}$ be the complex vector space of symmetric $\mathbb{R}$ bilinear maps $V \times V \rightarrow \mathbb{C}$. Elements of the latter space are (symmetric second rank complex-valued) tensors on $V$. Such a tensor $f \in S^{2} V_{\mathbb{C}}$ is identified with the symmetric operator $f: V_{\mathbb{C}} \rightarrow V_{\mathbb{C}}$. For a point $x \in \mathbb{R}^{n}$, by $x+V=\{x+v \mid v \in V\} \subset \mathbb{R}^{n}$ we denote the affine subspace (plane) through $x$ parallel to $V$. Let $\mathcal{S}\left(x+V ; S^{2} V_{\mathbb{C}}\right)$ be the Schwartz space of smooth fast decaying $S^{2} V_{\mathbb{C} \text {-valued }}$ functions on $x+V$. Elements of the space are (smooth fast decaying second rank) symmetric tensor fields on the plane $x+V$. The identical embedding $\iota: x+V \subset \mathbb{R}^{n}$ induces the linear operator 
$\iota^{*}: \mathcal{S}\left(\mathbb{R}^{n} ; S^{2} \mathbb{C}^{n}\right) \rightarrow \mathcal{S}\left(x+V ; S^{2} V_{\mathbb{C}}\right)$ by $\iota^{*} f(y)(v, w)=f(y)(v, w)$ for $y \in x+V$ and $v, w \in V$. For $f \in \mathcal{S}\left(\mathbb{R}^{n} ; S^{2} \mathbb{C}^{n}\right)$, the tensor field $\iota^{*} f \in \mathcal{S}\left(x+V ; S^{2} V_{\mathbb{C}}\right)$ will be called the slice of $f$ by the plane $x+V$. The Schwartz space $\mathcal{S}\left(x+V ; V_{\mathbb{C}}\right)$ of vector fields on $x+V$ is defined as well.

Besides the truncated transverse ray transform $K$ that has been introduced in the previous section, we need two other kinds of the ray transform. The transverse ray transform

$$
J: \mathcal{S}\left(\mathbb{R}^{n} ; S^{2} \mathbb{C}^{n}\right) \rightarrow \mathcal{S}\left(T \mathbb{S}^{n-1} ; S^{2} \mathbb{C}^{n}\right)
$$

is defined by

$$
J f(\xi, x)=\int_{-\infty}^{\infty} P_{\xi} f(x+t \xi) d t
$$

where $P_{\xi}: S^{2} \mathbb{C}^{n} \rightarrow S^{2} \mathbb{C}^{n}$ is the orthogonal projection onto the subspace $\left\{f \in S^{2} \mathbb{C}^{n} \mid f \xi=0\right\}$. The longitudinal ray transform

$$
I: \mathcal{S}\left(\mathbb{R}^{n} ; \mathbb{C}^{n}\right) \rightarrow \mathcal{S}\left(T \mathbb{S}^{n-1}\right), \quad I: \mathcal{S}\left(\mathbb{R}^{n} ; S^{2} \mathbb{C}^{n}\right) \rightarrow \mathcal{S}\left(T \mathbb{S}^{n-1}\right)
$$

is defined on vector and tensor fields by formulas

$$
I f(\xi, x)=\int_{-\infty}^{\infty}\langle f(x+t \xi), \xi\rangle d t \quad \text { and } \quad \operatorname{If}(\xi, x)=\int_{-\infty}^{\infty}\langle f(x+t \xi) \xi, \xi\rangle d t
$$

respectively.

The longitudinal ray transform can be also defined on a hyperplane of $\mathbb{R}^{n}$. For $\eta \in \mathbb{S}^{n-1}$, let $\eta^{\perp}=\left\{\xi \in \mathbb{R}^{n} \mid\langle\xi, \eta\rangle=0\right\}, \eta_{\mathbb{C}}^{\perp}$ be the complexification of $\eta^{\perp}$, and $\mathbb{S}_{\eta}^{n-2}=\left\{\xi \in \eta^{\perp}|| \xi \mid=1\right\}$ be the unit sphere in $\xi^{\perp}$. Given $s \in \mathbb{R}$, let $s \eta+\eta^{\perp}$ be the hyperplane through $s \eta$ parallel to $\eta^{\perp}$ and $\iota_{\eta, s}: s \eta+\eta^{\perp} \subset \mathbb{R}^{n}$ be the identical embedding. The family of oriented lines in the hyperplane $s \eta+\eta^{\perp}$ is parameterized by points of the manifold $T \mathbb{S}_{\eta}^{n-2}=\left\{(\xi, x) \mid \xi \in \mathbb{S}_{\eta}^{n-2}, x \in \eta^{\perp},\langle\xi, x\rangle=0\right\}$ such that a point $(\xi, x) \in T \mathbb{S}_{\eta}^{n-2}$ corresponds to the line $\{s \eta+x+t \xi \mid t \in \mathbb{R}\}$. The longitudinal ray transform on the hyperplane $s \eta+\eta^{\perp}$

$$
I_{\eta, s}: \mathcal{S}\left(s \eta+\eta^{\perp} ; \eta_{\mathbb{C}}^{\perp}\right) \rightarrow \mathcal{S}\left(T \mathbb{S}_{\eta}^{n-2}\right), \quad I_{\eta, s}: \mathcal{S}\left(s \eta+\eta^{\perp} ; S^{2} \eta_{\mathbb{C}}^{\perp}\right) \rightarrow \mathcal{S}\left(T \mathbb{S}_{\eta}^{n-2}\right)
$$

is defined on vector and tensor fields by formulas

$$
I_{\eta, s} f(\xi, x)=\int_{-\infty}^{\infty}\langle f(s \eta+x+t \xi), \xi\rangle d t \quad \text { and } \quad I_{\eta, s} f(\xi, x)=\int_{-\infty}^{\infty}\langle f(s \eta+x+t \xi) \xi, \xi\rangle d t
$$

respectively. Operators (3.1) and (3.2) are related as follows. If $f \in \mathcal{S}\left(\mathbb{R}^{n} ; S^{2} \mathbb{C}^{n}\right)$ and $\iota_{\eta, s}^{*} f$ is the slice of $f$ by the hyperplane $s \eta+\eta^{\perp}$, then $I_{\eta, s}\left(\iota_{\eta, s}^{*} f\right)(\xi, x)=I f(\xi, s \eta+x)$ for $(\xi, x) \in T \mathbb{S}_{\eta}^{n-2}$.

We will derive some relations between the three ray transforms. We will do this in the 3D case. For $\xi \in \mathbb{S}^{2}$ and a trace-free field $f \in \mathcal{S}\left(\mathbb{R}^{3} ; S^{2} \mathbb{C}^{3}\right)$, the projections $P_{\xi} f$ and $Q_{\xi} f$ are related by

$$
Q_{\xi} f(x)=P_{\xi} f(x)+\frac{1}{2}\langle f(x) \xi, \xi\rangle \varepsilon(\xi),
$$

where $\varepsilon_{i j}(\xi)=\delta_{i j}-\xi_{i} \xi_{j}$. Formula (3.3) is a partial case of Lemma 6.4.2 of [11]. Setting $x:=x+t \xi$ in (3.3) and integrating the result over $t$, we obtain

$$
(K f)(\xi, x)=(J f)(\xi, x)+\frac{1}{2}(I f)(\xi, x) \cdot \varepsilon(\xi) \quad \text { for } \quad(\xi, x) \in T \mathbb{S}^{2} .
$$

Let a vector $\eta \in \mathbb{S}^{2}$ be orthogonal to $\xi$. Then $\varepsilon(\xi) \eta=\eta$ and formula (3.4) gives

$$
(K f)(\xi, x) \eta=(J f)(\xi, x) \eta+\frac{1}{2}(I f)(\xi, x) \eta \quad \text { for }(\xi, x) \in T \mathbb{S}^{2},\langle\xi, \eta\rangle=0,|\eta|=1 .
$$


Multiply equation $(3.5)$ by $\xi \times \eta$

$$
\langle(K f)(\xi, x) \eta, \xi \times \eta\rangle=\langle(J f)(\xi, x) \eta, \xi \times \eta\rangle \quad \text { for }(\xi, x) \in T \mathbb{S}^{2},\langle\xi, \eta\rangle=0,|\eta|=1 .
$$

The right-hand side of the latter equation can be transformed as follows. As is easily seen, $P_{\xi} f=$ $\pi_{\xi} f \pi_{\xi}$ where $\pi_{\xi}: \mathbb{R}^{3} \rightarrow \mathbb{R}^{3}$ is the orthogonal projection onto $\xi^{\perp}$. Since $\pi_{\xi} \eta=\eta$ and $\pi_{\xi}(\xi \times \eta)=\xi \times \eta$,

$$
\begin{aligned}
\langle(J f)(\xi, x) \eta, \xi \times \eta\rangle & =\int_{-\infty}^{\infty}\left\langle\pi_{\xi} f(x+t \xi) \pi_{\xi} \eta, \xi \times \eta\right\rangle d t \\
& =\int_{-\infty}^{\infty}\langle f(x+t \xi) \eta, \xi \times \eta\rangle d t=\int_{-\infty}^{\infty}\langle\eta \times f(x+t \xi) \eta, \xi\rangle d t .
\end{aligned}
$$

Equation (3.6) takes the form

$$
\langle(K f)(\xi, x) \eta, \xi \times \eta\rangle=\int_{-\infty}^{\infty}\langle\eta \times f(x+t \xi) \eta, \xi\rangle d t \quad \text { for }(\xi, x) \in T \mathbb{S}^{2},\langle\xi, \eta\rangle=0,|\eta|=1 .
$$

Since the vector field $\eta \times f \eta$ is orthogonal to $\eta$, its restriction to every plane $s \eta+\eta^{\perp}$ can be considered as the $2 \mathrm{D}$ vector field on the plane, i.e., $\left.(\eta \times f \eta)\right|_{s \eta+\eta^{\perp}} \in \mathcal{S}\left(s \eta+\eta^{\perp} ; \eta_{\mathbb{C}}^{\perp}\right)$. Formula (3.7) means that

$$
I_{\eta, s}\left(\left.(\eta \times f \eta)\right|_{s \eta+\eta^{\perp}}\right)(\xi, x)=\langle(K f)(\xi, s \eta+x) \eta, \xi \times \eta\rangle \quad \text { for } \quad(\xi, x) \in T \mathbb{S}_{\eta}^{1} .
$$

Now, we multiply equation $(3.5)$ by $\eta$

$$
\langle(K f)(\xi, x) \eta, \eta\rangle=\langle(J f)(\xi, x) \eta, \eta\rangle+\frac{1}{2}(I f)(\xi, x) \text { for }(\xi, x) \in T \mathbb{S}^{2},\langle\xi, \eta\rangle=0,|\eta|=1 .
$$

The first term on the right-hand side of (3.8) can be transformed as follows:

$$
\langle(J f)(\xi, x) \eta, \eta\rangle=\int_{-\infty}^{\infty}\left\langle\pi_{\xi} f(x+t \xi) \eta, \eta\right\rangle d t=\int_{-\infty}^{\infty}\left\langle f(x+t \xi) \eta, \pi_{\xi} \eta\right\rangle d t=\int_{-\infty}^{\infty}\langle f(x+t \xi) \eta, \eta\rangle d t .
$$

Formula (3.8) becomes

$$
\langle(K f)(\xi, x) \eta, \eta\rangle=\int_{-\infty}^{\infty}\langle f(x+t \xi) \eta, \eta\rangle d t+\frac{1}{2}(I f)(x, \xi) \text { for }(\xi, x) \in T \mathbb{S}^{2},\langle\xi, \eta\rangle=0,|\eta|=1 .
$$

For a fixed unit vector $\eta \in \mathbb{R}^{3}$ and $s \in \mathbb{R}$, we define the function $\varphi$ on the plane $s \eta+\eta^{\perp}$ by $\varphi(x)=\langle f(x) \eta, \eta\rangle$. Formula (3.9) means that

$$
I_{\eta, s}\left(\iota_{\eta, s}^{*} f+2 \varphi \delta\right)(\xi, x)=2\langle(K f)(\xi, s \eta+x) \eta, \eta\rangle \quad \text { for } \quad(\xi, x) \in T \mathbb{S}_{\eta}^{1},
$$

where $\delta$ is the Kronecker tensor on $\xi^{\perp}$.

The function $\varphi$ can be expressed through the tensor $\iota_{\eta, s}^{*} f$. Indeed, let $\left(\xi, \xi^{\prime}, \eta\right)$ be the orthonormal basis of $\mathbb{R}^{3}$. Then

The three last formulas imply

$$
\begin{gathered}
0=\operatorname{tr} f(x)=\langle f(x) \xi, \xi\rangle+\left\langle f(x) \xi^{\prime}, \xi^{\prime}\right\rangle+\langle f(x) \eta, \eta\rangle, \\
\varphi(x)=\langle f(x) \eta, \eta\rangle \text { for } \quad x \in s \eta+\eta^{\perp}, \\
\operatorname{tr} \iota_{\eta, s}^{*} f(x)=\langle f(x) \xi, \xi\rangle+\left\langle f(x) \xi^{\prime}, \xi^{\prime}\right\rangle \text { for } x \in s \eta+\eta^{\perp} .
\end{gathered}
$$

$$
\varphi(x)=-\operatorname{tr} \iota_{\eta, s}^{*} f(x) \text { for } \quad x \in s \eta+\eta^{\perp} .
$$

Substituting this value into (3.10), we obtain

$$
I_{\eta, s}\left(\iota_{\eta, s}^{*} f-2\left(\operatorname{tr} \iota_{\eta, s}^{*} f\right) \delta\right)(\xi, x)=2\langle(K f)(\xi, s \eta+x) \eta, \eta\rangle \quad \text { for } \quad(\xi, x) \in T \mathbb{S}_{\eta}^{1} .
$$


We summarize results of this section in the following statement.

LEMMA 3.1. Let $f \in \mathcal{S}\left(\mathbb{R}^{3} ; S^{2} \mathbb{C}^{3}\right)$ be a trace-free tensor field. Equations

$$
\begin{gathered}
I_{\eta, s}\left(\left.(\eta \times f \eta)\right|_{s \eta+\eta^{\perp}}\right)=K_{\eta, s}^{1} f, \\
I_{\eta, s}\left(\iota_{\eta, s}^{*} f-2 \operatorname{tr}\left(\iota_{\eta, s}^{*} f\right) \delta\right)=2 K_{\eta, s}^{2} f
\end{gathered}
$$

hold for every $s \in \mathbb{R}$ and $\eta \in \mathbb{S}^{2}$, where the functions $K_{\eta, s}^{i} f \in \mathcal{S}\left(T \mathbb{S}_{\eta}^{1}\right)(i=1,2)$ are defined by

$$
\left(K_{\eta, s}^{1} f\right)(\xi, x)=\langle(K f)(\xi, s \eta+x) \eta, \xi \times \eta\rangle, \quad\left(K_{\eta, s}^{2} f\right)(\xi, x)=\langle(K f)(\xi, s \eta+x) \eta, \eta\rangle .
$$

For $\eta \in \mathbb{S}^{2}$, the submanifold $L_{\eta}^{3} \subset T \mathbb{S}^{2}$ was defined in Section 2 . We identify $L_{\eta}^{3}$ with $\mathbb{R} \times T \mathbb{S}_{\eta}^{1}$ by the diffeomorphism $\mathbb{R} \times T \mathbb{S}_{\eta}^{1} \rightarrow L_{\eta}^{3},(s ; \xi, x) \mapsto(\xi, s \eta+x)$. As was mentioned in Section 2 , the matrix function $\left.K f\right|_{L_{\eta}^{3}}$ has two linearly independent components. The functions $K_{\eta}^{i} f \in \mathcal{S}\left(\mathbb{R} \times T \mathbb{S}_{\eta}^{1}\right)=$ $\mathcal{S}(\mathbb{R}) \otimes \mathcal{S}\left(T \mathbb{S}_{\eta}^{1}\right)(i=1,2)$

$$
\left(K_{\eta}^{1} f\right)(s ; \xi, x)=\langle(K f)(\xi, s \eta+x) \eta, \xi \times \eta\rangle, \quad\left(K_{\eta}^{2} f\right)(s ; \xi, x)=\langle(K f)(\xi, s \eta+x) \eta, \eta\rangle
$$

are just such independent components. The function $K_{\eta, s}^{i} f$ from Lemma 3.1 is actually the $s$-slice of $K_{\eta}^{i} f$, i.e., $\left(K_{\eta, s}^{i} f\right)(\xi, x)=K_{\eta}^{i}(s ; \xi, x)$. Thus, given the data $\left.K f\right|_{L_{\eta}^{3}}$ for a trace-free tensor field $f \in \mathcal{S}\left(\mathbb{R}^{3} ; S^{2} \mathbb{C}^{3}\right)$, right-hand sides of (3.11)-(3.12) are known for every $s$, and we can consider (3.11) and (3.12) as linear equations with the unknown $f$. In the next section, we will transform (3.11)-(3.12) to algebraic equations by applying the Fourier transform.

\section{Main algebraic equations}

First of all, we briefly recall inversion formulas for $2 \mathrm{D}$ vector and tensor fields which recover the tangential component of $\hat{f}$ from $I f$. See Sections 2.4 and 3.4 of [12] for details.

The tangential component $\tau g \in C^{\infty}\left(\mathbb{R}^{2}\right)$ of a vector field $g \in C^{\infty}\left(\mathbb{R}^{2} ; \mathbb{C}^{2}\right)$ is defined by

$$
(\tau g)(y)=\left\langle g(y), y^{\perp}\right\rangle \text {, }
$$

see formula $(3.8)$ of $[\mathbf{1 2}]$. Here the vector $y^{\perp}$ is the result of rotating $y$ by $\pi / 2$ in the positive direction, $\mathbb{R}^{2}$ being endowed the standard orientation.

The manifold $T \mathbb{S}^{1}$ can be identified with $\mathbb{R} \times \mathbb{S}^{1}$ by the diffeomorphism $(p, \xi) \mapsto\left(\xi, p \xi^{\perp}\right)$ for $(p, \xi) \in \mathbb{R} \times \mathbb{S}^{1}$. Therefore the derivative $\frac{\partial}{\partial p}: \mathcal{S}\left(T \mathbb{S}^{1}\right) \rightarrow \mathcal{S}\left(T \mathbb{S}^{1}\right)$ is well defined.

For a vector field $f \in \mathcal{S}\left(\mathbb{R}^{2} ; \mathbb{C}^{2}\right)$, the tangential component of the Fourier transform $F[f]$ is recovered from the ray transform If by the formula

$$
\tau F[f]=\frac{i}{2}|y| F\left[B\left(\frac{\partial(I f)}{\partial p}\right)\right],
$$

see formula $(2.24)$ of $[\mathbf{1 2}]$. Here $B: \mathcal{S}\left(T \mathbb{S}^{1}\right) \rightarrow C^{\infty}\left(\mathbb{R}^{2}\right)$ is the back projection operator. If $T \mathbb{S}^{1}$ is considered as the manifold of oriented lines in $\mathbb{R}^{2}$ then, for $\varphi \in \mathcal{S}\left(T \mathbb{S}^{1}\right)$, the value of the function $B \varphi$ at a point $x \in \mathbb{R}^{2}$ is just the average of $\varphi$ over all lines passing through $x$. The analytical expression for $B$ can be written as follows. Choose an orthonormal basis $\left(e_{1}, e_{2}\right)$ of $\mathbb{R}^{2}$ and let $\xi(\theta)=\cos \theta e_{1}+\sin \theta e_{2}$ and $\xi^{\perp}(\theta)=-\sin \theta e_{1}+\cos \theta e_{2}$. Then

$$
(B \varphi)(x)=\frac{1}{2 \pi} \int_{0}^{2 \pi} \varphi\left(\xi(\theta),\left\langle x, \xi^{\perp}(\theta)\right\rangle \xi^{\perp}(\theta)\right) d \theta .
$$

The tangential component $\tau g \in C^{\infty}\left(\mathbb{R}^{2}\right)$ of a tensor field $g \in C^{\infty}\left(\mathbb{R}^{2} ; S^{2} \mathbb{C}^{2}\right)$ can be defined by

$$
(\tau g)(y)=|y|^{2} \operatorname{tr} g-\langle g(y) y, y\rangle
$$


see formula (3.8) of [12]. For $f \in \mathcal{S}\left(\mathbb{R}^{2} ; S^{2} \mathbb{C}^{2}\right)$, the tangential component of the Fourier transform $F[f]$ is recovered from the ray transform $I f$ by the formula

$$
\tau F[f]=\frac{1}{2}|y|^{3} F[B(I f)],
$$

see formula $(3.19)$ of $[\mathbf{1 2}]$.

For $\varphi \in \mathcal{S}\left(T \mathbb{S}^{1}\right)$, the function $B \varphi(x)$ is $C^{\infty}$-smooth and bounded on $\mathbb{R}^{2}$ but does not fast decay as $|x| \rightarrow \infty$. Therefore the Fourier transform on right-hand sides of (4.2) and (4.5) is understood in the distribution sense.

We return to the 3D problem. Let $f \in \mathcal{S}\left(\mathbb{R}^{3} ; S^{2} \mathbb{C}^{3}\right)$ be a trace-free tensor field. Fix a unit vector $\eta \in \mathbb{S}^{2}$ and denote by $f^{\prime}=F_{\eta^{\perp}}[f] \in \mathcal{S}\left(\mathbb{R}^{3} ; S^{2} \mathbb{C}^{3}\right)$ the partial Fourier transform of $f$. For any $s \in \mathbb{R}$, the restriction of the vector field $\eta \times f^{\prime} \eta$ to the plane $s \eta+\eta^{\perp}$ coincides with the 2D Fourier transform of $\left.(\eta \times f \eta)\right|_{s \eta+\eta^{\perp}}$, i.e., $\left.\left(\eta \times f^{\prime} \eta\right)\right|_{s \eta+\eta^{\perp}}=F\left[\left.(\eta \times f \eta)\right|_{s \eta+\eta^{\perp}}\right]$, where $F$ is the Fourier transform on the plane $s \eta+\eta^{\perp}$. Apply formula (4.2) to the vector field $\left.(\eta \times f \eta)\right|_{s \eta+\eta^{\perp}} \in \mathcal{S}\left(s \eta+\eta^{\perp} ; \eta_{\mathbb{C}}^{\perp}\right)$

$$
\tau\left(\left.\left(\eta \times f^{\prime} \eta\right)\right|_{s \eta+\eta^{\perp}}\right)(s \eta+y)=\frac{i}{2}|y| F_{\eta^{\perp}}\left[B_{\eta}\left(\frac{\partial\left(I_{\eta, s}\left(\left.(\eta \times f \eta)\right|_{s \eta+\eta^{\perp}}\right)\right)}{\partial p}\right)\right] \text { for } y \in \eta^{\perp},
$$

where $B_{\eta}: \mathcal{S}\left(T \mathbb{S}_{\eta}^{1}\right) \rightarrow C^{\infty}\left(\eta^{\perp}\right)$ is the back projection operator on the plane $\eta^{\perp}$. Comparing the right-hand side of this formula with (3.11) and (3.13), we obtain

$$
\tau\left(\left.\left(\eta \times f^{\prime} \eta\right)\right|_{s \eta+\eta^{\perp}}\right)(s \eta+y)=\frac{i}{2}|y| F_{x \rightarrow y}\left[\left(B_{\eta} \frac{\partial\left(K_{\eta}^{1} f\right)}{\partial p}\right)(s \eta+x)\right] \text { for } y \in \eta^{\perp},
$$

where $F_{x \rightarrow y}$ is the Fourier transform on the plane $\eta^{\perp}$.

By (4.1),

$$
\tau\left(\left.\left(\eta \times f^{\prime} \eta\right)\right|_{s \eta+\eta^{\perp}}\right)(s \eta+y)=\left\langle\eta \times f^{\prime}(s \eta+y) \eta, \eta \times y\right\rangle=\left\langle f^{\prime}(s \eta+y) \eta, y\right\rangle .
$$

Formula (4.6) takes the form

$$
\left\langle f^{\prime}(s \eta+y) \eta, y\right\rangle=\frac{i}{2}|y| F_{x \rightarrow y}\left[\left(B_{\eta} \frac{\partial\left(K_{\eta}^{1} f\right)}{\partial p}\right)(s \eta+x)\right] \text { for } y \in \eta^{\perp} .
$$

Applying the one-dimensional Fourier transform $F_{s \rightarrow \sigma}$ to this equation, we obtain

$$
\left\langle\hat{f}\left(\sigma \eta+y^{\prime}\right) \eta, y^{\prime}\right\rangle=\frac{i}{2}\left|y^{\prime}\right| F_{s \eta+x^{\prime} \rightarrow \sigma \eta+y^{\prime}}\left[\left(B_{\eta} \frac{\partial\left(K_{\eta}^{1} f\right)}{\partial p}\right)\left(s \eta+x^{\prime}\right)\right] \text { for } y^{\prime} \in \eta^{\perp},
$$

where $\hat{f}$ is the 3D Fourier transform of $f$. If, for $y^{\prime} \in \eta^{\perp}$ and $\sigma \in \mathbb{R}$, let $y=\sigma \eta+y^{\prime}$, then $y^{\prime}=\pi_{\eta} y$ where $\pi_{\eta}: \mathbb{R}^{3} \rightarrow \mathbb{R}^{3}$ is the orthogonal projection onto $\eta^{\perp}$. Therefore the last formula can be written as

$$
\left\langle\hat{f}(y) \eta, \pi_{\eta} y\right\rangle=\frac{i}{2}\left|\pi_{\eta} y\right| F_{x \rightarrow y}\left[\left(B_{\eta} \frac{\partial\left(K_{\eta}^{1} f\right)}{\partial p}\right)(x)\right],
$$

where $F_{x \rightarrow y}$ is the 3D Fourier transform. The back projection is now understood as the linear operator $B_{\eta}: \mathcal{S}\left(\mathbb{R} \times T \mathbb{S}_{\eta}^{1}\right) \rightarrow C^{\infty}\left(\mathbb{R}^{3}\right)$ defined by

$$
\left(B_{\eta} \varphi\right)(s \eta+x)=\frac{1}{2 \pi} \int_{0}^{2 \pi} \varphi\left(s ; \xi(\theta),\left\langle x, \xi^{\perp}(\theta)\right\rangle \xi^{\perp}(\theta)\right) d \theta \quad \text { for } \quad x \in \eta^{\perp},
$$

where $\xi(\theta)=\cos \theta e_{1}+\sin \theta e_{2}$ and $\xi^{\perp}(\theta)=-\sin \theta e_{1}+\cos \theta e_{2}$ with respect to an orthonormal basis $\left(e_{1}, e_{2}\right)$ of $\eta^{\perp}$. The operator has the obvious sense: the value of $B_{\eta} \varphi$ at a point $x \in \mathbb{R}^{3}$ is just the average of the function $\varphi$ over all lines passing through $x$ and orthogonal to $\eta$.

Let again $\eta \in \mathbb{S}^{2}$ be a fixed vector and $f^{\prime}=F_{\eta^{\perp}}[f]$ be the partial Fourier transform of a tracefree tensor field $f \in \mathcal{S}\left(\mathbb{R}^{3} ; S^{2} \mathbb{C}^{3}\right)$. For any $s \in \mathbb{R}$, the slice $\iota_{\eta, s}^{*} f^{\prime}$ coincides with the $2 \mathrm{D}$ Fourier 
transform of the slice $\iota_{\eta, s}^{*} f$, i.e., $\iota_{\eta, s}^{*} f^{\prime}=F\left[\iota_{\eta, s}^{*} f\right]$, where $F$ is the Fourier transform on the plane $s \eta+\eta^{\perp}$. Apply formula (4.5) to the tensor field $\iota_{\eta, s}^{*} f-2 \operatorname{tr}\left(\iota_{\eta, s}^{*} f\right) \delta \in \mathcal{S}\left(s \eta+\eta^{\perp} ; S^{2} \eta_{\mathbb{C}}^{\perp}\right)$

$$
\left[\tau\left(\iota_{\eta, s}^{*} f^{\prime}-2 \operatorname{tr}\left(\iota_{\eta, s}^{*} f^{\prime}\right) \delta\right)\right](s \eta+y)=\frac{1}{2}|y|^{3} F_{\eta^{\perp}}\left[B_{\eta}\left(I_{\eta, s}\left(\iota_{\eta, s}^{*} f-2 \operatorname{tr}\left(\iota_{\eta, s}^{*} f\right) \delta\right)\right)\right] \quad \text { for } y \in \eta^{\perp} .
$$

Comparing the right-hand side of this formula with (3.12)-(3.13), we obtain

$$
\left[\tau\left(\iota_{\eta, s}^{*} f^{\prime}-2 \operatorname{tr}\left(\iota_{\eta, s}^{*} f^{\prime}\right) \delta\right)\right](s \eta+y)=|y|^{3} F_{x \rightarrow y}\left[\left(B_{\eta} K_{\eta}^{2} f\right)(s \eta+x)\right] \quad \text { for } \quad y \in \eta^{\perp},
$$

where $F_{x \rightarrow y}$ is the Fourier transform on the plane $\eta^{\perp}$.

Now, we are going to express the left-hand side of (4.9) in terms of $f^{\prime}$. First of all, since $(\tau \delta)(s \eta+y)=|y|^{2}$,

$$
\left[\tau\left(\iota_{\eta, s}^{*} f^{\prime}-2 \operatorname{tr}\left(\iota_{\eta, s}^{*} f^{\prime}\right) \delta\right)\right](s \eta+y)=\left[\tau\left(\iota_{\eta, s}^{*} f^{\prime}\right)-2|y|^{2} \operatorname{tr}\left(\iota_{\eta, s}^{*} f^{\prime}\right)\right](s \eta+y) \quad \text { for } \quad y \in \eta^{\perp} .
$$

Apply formula (4.4) to the field $g=\iota_{\eta, s}^{*} f^{\prime} \in \mathcal{S}\left(s \eta+\eta^{\perp} ; S^{2} \eta_{\mathbb{C}}^{\perp}\right)$

$$
\left[\tau\left(\iota_{\eta, s}^{*} f^{\prime}\right)\right](s \eta+y)=|y|^{2} \operatorname{tr}\left(\iota_{\eta, s}^{*} f^{\prime}\right)-\left\langle\left(\iota_{\eta, s}^{*} f^{\prime}\right)(s \eta+y) y, y\right\rangle \quad \text { for } \quad y \in \eta^{\perp} .
$$

By the definition of the slice, $\left\langle\left(\iota_{\eta, s}^{*} f^{\prime}\right)(s \eta+y) y, y\right\rangle=\left\langle f^{\prime}(s \eta+y) y, y\right\rangle$ for $y \in \eta^{\perp}$. Therefore the last formula is simplified to the following one:

$$
\left[\tau\left(\iota_{\eta, s}^{*} f^{\prime}\right)\right](s \eta+y)=|y|^{2} \operatorname{tr}\left(\iota_{\eta, s}^{*} f^{\prime}\right)-\left\langle f^{\prime}(s \eta+y) y, y\right\rangle \quad \text { for } \quad y \in \eta^{\perp} .
$$

Substitute this expression into (4.10)

(4.11) $\left[\tau\left(\iota_{\eta, s}^{*} f^{\prime}-2 \operatorname{tr}\left(\iota_{\eta, s}^{*} f^{\prime}\right) \delta\right)\right](s \eta+y)=-|y|^{2} \operatorname{tr}\left(\iota_{\eta, s}^{*} f^{\prime}\right)(s \eta+y)-\left\langle f^{\prime}(s \eta+y) y, y\right\rangle \quad$ for $\quad y \in \eta^{\perp}$.

If $\left(e_{1}, e_{2}\right)$ is an orthonormal basis of $\eta^{\perp}$, then

This implies

$$
\begin{gathered}
0=\operatorname{tr} f^{\prime}=\left\langle f^{\prime} e_{1}, e_{1}\right\rangle+\left\langle f^{\prime} e_{2}, e_{2}\right\rangle+\left\langle f^{\prime} \eta, \eta\right\rangle, \\
\operatorname{tr}\left(\iota_{s \eta}^{*} f^{\prime}\right)=\left\langle f^{\prime} e_{1}, e_{1}\right\rangle+\left\langle f^{\prime} e_{2}, e_{2}\right\rangle .
\end{gathered}
$$

$$
\operatorname{tr}\left(\iota_{s \eta}^{*} f^{\prime}\right)=-\left\langle f^{\prime} \eta, \eta\right\rangle
$$

With the help of the last equation, formula (4.11) takes the final form

$$
\left[\tau\left(\iota_{\eta, s}^{*} f^{\prime}-2 \operatorname{tr}\left(\iota_{\eta, s}^{*} f^{\prime}\right) \delta\right)\right](s \eta+y)=|y|^{2}\left\langle f^{\prime}(s \eta+y) \eta, \eta\right\rangle-\left\langle f^{\prime}(s \eta+y) y, y\right\rangle \quad \text { for } \quad y \in \eta^{\perp} .
$$

Substitute (4.12) into (4.9)

$$
|y|^{2}\left\langle f^{\prime}(s \eta+y) \eta, \eta\right\rangle-\left\langle f^{\prime}(s \eta+y) y, y\right\rangle=|y|^{3} F_{x \rightarrow y}\left[\left(B_{\eta} K_{\eta}^{2} f\right)(s \eta+x)\right] \quad \text { for } \quad y \in \eta^{\perp} .
$$

Applying the one-dimensional Fourier transform $F_{s \rightarrow \sigma}$ to this equation, we obtain

$$
\left|y^{\prime}\right|^{2}\left\langle\hat{f}\left(\sigma \eta+y^{\prime}\right) \eta, \eta\right\rangle-\left\langle\hat{f}\left(\sigma \eta+y^{\prime}\right) y^{\prime}, y^{\prime}\right\rangle=\left|y^{\prime}\right|^{3} F_{s \eta+x^{\prime} \rightarrow \sigma \eta+y^{\prime}}\left[\left(B_{\eta} K_{\eta}^{2} f\right)\left(s \eta+x^{\prime}\right)\right]
$$

for $y^{\prime} \in \eta^{\perp}$. Changing the variables $y=\sigma \eta+y^{\prime}$ as before, we write this equation in the form

$$
\left|\pi_{\eta} y\right|^{2}\langle\hat{f}(y) \eta, \eta\rangle-\left\langle\hat{f}(y) \pi_{\eta} y, \pi_{\eta} y\right\rangle=\left|\pi_{\eta} y\right|^{3} F_{x \rightarrow y}\left[\left(B_{\eta} K_{\eta}^{2} f\right)(x)\right] \quad \text { for } \quad y \in \mathbb{R}^{3} .
$$

We summarize results of this section in the following statement.

Lemma 4.1. Let $\hat{f}$ be the $3 D$ Fourier transform of a trace-free tensor field $f \in \mathcal{S}\left(\mathbb{R}^{3} ; S^{2} \mathbb{C}^{3}\right)$. For a unit vector $\eta \in \mathbb{S}^{2}$, equations

$$
\left\langle\hat{f}(y) \eta, \pi_{\eta} y\right\rangle=\lambda_{\eta}(y)
$$

and

$$
\left|\pi_{\eta} y\right|^{2}\langle\hat{f}(y) \eta, \eta\rangle-\left\langle\hat{f}(y) \pi_{\eta} y, \pi_{\eta} y\right\rangle=\mu_{\eta}(y)
$$

hold on $\mathbb{R}^{3}$ with right-hand sides defined by

$$
\lambda_{\eta}(y)=\frac{i}{2}\left|\pi_{\eta} y\right| F_{x \rightarrow y}\left[\left(B_{\eta} \frac{\partial\left(K_{\eta}^{1} f\right)}{\partial p}\right)(x)\right],
$$




$$
\mu_{\eta}(y)=\left|\pi_{\eta} y\right|^{3} F_{x \rightarrow y}\left[\left(B_{\eta} K_{\eta}^{2} f\right)(x)\right] .
$$

Here $\pi_{\eta}: \mathbb{R}^{3} \rightarrow \mathbb{R}^{3}$ is the orthogonal projection onto $\eta^{\perp}, F_{x \rightarrow y}$ is the $3 D$ Fourier transform on $\mathbb{R}^{3}$, the back projection operator $B_{\eta}: \mathcal{S}\left(\mathbb{R} \times T \mathbb{S}_{\eta}^{1}\right) \rightarrow C^{\infty}\left(\mathbb{R}^{3}\right)$ is defined by (4.8), and operators $K_{\eta}^{i}: \mathcal{S}\left(\mathbb{R}^{3} ; S^{2} \mathbb{C}^{3}\right) \rightarrow \mathcal{S}\left(\mathbb{R} \times T \mathbb{S}_{\eta}^{1}\right)(i=1,2)$ are defined by

$$
\begin{gathered}
\left(K_{\eta}^{1} f\right)(s ; \xi, x)=\langle(K f)(\xi, s \eta+x) \eta, \xi \times \eta\rangle \quad \text { for } \quad s \in \mathbb{R} \quad \text { and } \quad(\xi, x) \in T \mathbb{S}_{\eta}^{1}, \\
\left(K_{\eta}^{2} f\right)(s ; \xi, x)=\langle(K f)(\xi, s \eta+x) \eta, \eta\rangle \quad \text { for } \quad s \in \mathbb{R} \quad \text { and } \quad(\xi, x) \in T \mathbb{S}_{\eta}^{1},
\end{gathered}
$$

$K$ being the truncated transverse ray transform. The partial derivative $\frac{\partial}{\partial p}: \mathcal{S}\left(\mathbb{R} \times T \mathbb{S}_{\eta}^{1}\right) \rightarrow \mathcal{S}\left(\mathbb{R} \times T \mathbb{S}_{\eta}^{1}\right)$ is defined with the help of the diffeomorphism $\mathbb{R}^{2} \times \mathbb{S}_{\eta}^{1} \rightarrow \mathbb{R} \times T \mathbb{S}_{\eta}^{1},(s, p, \xi) \mapsto(s, \xi, p \xi \times \eta)$.

Given the data $\left.K f\right|_{L_{\eta}^{3}}$, right-hand sides $\lambda_{\eta}(y)$ and $\mu_{\eta}(y)$ of equations (4.13)-(4.14) can be effectively recovered by formulas (4.15)-(4.18). Thus, (4.13) and (4.14) are linear algebraic equations in unknowns $\hat{f}_{j k}(y)$ with known right-hand sides and with coefficients depending linearly (quadratically) on $y$.

In next two sections, we will investigate systems obtained by combining equations (4.13)-(4.14) for several values of $\eta$. Only the data $\left(\lambda_{\eta}, \mu_{\eta}\right)$ are used for each value of $\eta$ in what follows.

\section{System for three directions}

Here, we are going to investigate the system obtained by combining equations (4.13)-(4.14) for vectors $\left(\eta_{1}, \eta_{2}, \eta_{3}\right)$ constituting an orthonormal basis of $\mathbb{R}^{3}$. To abbreviate further formulas, let us denote $\lambda_{\eta_{i}}$ by $\lambda_{i}$ and $\mu_{\eta_{i}}$ by $\lambda_{i}$.

For $\eta=\eta_{1}=(1,0,0)$, we have $\pi_{\eta} y=\left(0, y_{2}, y_{3}\right), \hat{f} \eta=\left(\hat{f}_{11}, \hat{f}_{12}, \hat{f}_{13}\right)$ and

$$
\left|\pi_{\eta} y\right|^{2}\langle\hat{f} \eta, \eta\rangle-\left\langle\hat{f} \pi_{\eta} y, \pi_{\eta} y\right\rangle=\left(y_{2}^{2}+y_{3}^{2}\right) \hat{f}_{11}-\left(\hat{f}_{22} y_{2}^{2}+2 \hat{f}_{23} y_{2} y_{3}+\hat{f}_{33} y_{3}^{2}\right) .
$$

Using the relation $\operatorname{tr} \hat{f}=0$, this can be written as

$$
\left|\pi_{\eta} y\right|^{2}\langle\hat{f} \eta, \eta\rangle-\left\langle\hat{f} \pi_{\eta} y, \pi_{\eta} y\right\rangle=-\left(\left(2 y_{2}^{2}+y_{3}^{2}\right) \hat{f}_{22}+2 y_{2} y_{3} \hat{f}_{23}+\left(y_{2}^{2}+2 y_{3}^{2}\right) \hat{f}_{33}\right) .
$$

Therefore equations (4.13)-(4.14) for $\eta_{1}$ are as follows:

$$
\begin{gathered}
y_{2} \hat{f}_{12}+y_{3} \hat{f}_{13}=\lambda_{1} . \\
\left(2 y_{2}^{2}+y_{3}^{2}\right) \hat{f}_{22}+2 y_{2} y_{3} \hat{f}_{23}+\left(y_{2}^{2}+2 y_{3}^{2}\right) \hat{f}_{33}=-\mu_{1} .
\end{gathered}
$$

The corresponding equations for $\eta_{2}$ and $\eta_{3}$ are obtained from (5.1)-(5.2) by cyclic transposing indices. In such the way we arrive to the systems

$$
\left\{\begin{array}{l}
y_{2} \hat{f}_{12}+y_{3} \hat{f}_{13}=\lambda_{1}, \\
y_{1} \hat{f}_{12}+y_{3} \hat{f}_{23}=\lambda_{2}, \\
y_{1} \hat{f}_{13}+y_{2} \hat{f}_{23}=\lambda_{3} \\
\left(2 y_{2}^{2}+y_{3}^{2}\right) \hat{f}_{22}+2 y_{2} y_{3} \hat{f}_{23}+\left(y_{2}^{2}+2 y_{3}^{2}\right) \hat{f}_{33}=-\mu_{1}, \\
\left(2 y_{1}^{2}+y_{3}^{2}\right) \hat{f}_{11}+2 y_{1} y_{3} \hat{f}_{13}+\left(y_{1}^{2}+2 y_{3}^{2}\right) \hat{f}_{33}=-\mu_{2}, \\
\left(2 y_{1}^{2}+y_{2}^{2}\right) \hat{f}_{11}+2 y_{1} y_{2} \hat{f}_{12}+\left(y_{1}^{2}+2 y_{2}^{2}\right) \hat{f}_{22}=-\mu_{3} .
\end{array}\right.
$$

One can easily check that system (5.3) is nondegenerate at every point $y \in \mathbb{R}^{3}$ which does not belong to any coordinate plane. Thus, the system is uniquely solvable for almost all $y$. By continuity, $\hat{f}(y)$ is uniquely determined on the whole of $\mathbb{R}^{3}$. This gives the uniqueness statement: a trace-free tensor field $f \in \mathcal{S}\left(\mathbb{R}^{3} ; S^{2} \mathbb{C}^{3}\right)$ is uniquely determined by the data $\left(\left.K f\right|_{L_{\eta_{1}}^{3}},\left.K f\right|_{L_{\eta_{2}}^{3}},\left.K f\right|_{L_{\eta_{3}}^{3}}\right)$ for an orthonormal basis $\left(\eta_{1}, \eta_{2}, \eta_{3}\right)$. 
Substituting the solution to system (5.3) into the equation $\operatorname{tr} \hat{f}=0$, we obtain the relation

$$
y_{1}\left(-y_{1}^{2}+y_{2}^{2}+y_{3}^{2}\right) \lambda_{1}+y_{2}\left(y_{1}^{2}-y_{2}^{2}+y_{3}^{2}\right) \lambda_{2}+y_{3}\left(y_{1}^{2}+y_{2}^{2}-y_{3}^{2}\right) \lambda_{3}+y_{1}^{2} \mu_{1}+y_{2}^{2} \mu_{2}+y_{3}^{2} \mu_{3}=0
$$

which can be considered as a consistency condition.

Let us prove the more general statement: a trace-free tensor field $f$ is uniquely determined by the data $\left(\left.K f\right|_{L_{\eta_{1}}^{3}},\left.K f\right|_{L_{\eta_{2}}^{3}},\left.K f\right|_{L_{\eta_{3}}^{3}}\right)$ for a generic family $\left(\eta_{1}, \eta_{2}, \eta_{3}\right)$ of three unit vectors. To this end we introduce the functions

$$
\begin{gathered}
a(\eta, y, \hat{f})=\left\langle\hat{f} \eta,|\eta|^{2} y-\langle y, \eta\rangle \eta\right\rangle \\
b(\eta, y, \hat{f})=\left.|| \eta\right|^{2} y-\left.\langle y, \eta\rangle \eta\right|^{2}\langle\hat{f} \eta, \eta\rangle-|\eta|^{2}\left\langle\hat{f}\left(|\eta|^{2} y-\langle y, \eta\rangle \eta\right),|\eta|^{2} y-\langle y, \eta\rangle \eta\right\rangle
\end{gathered}
$$

They are polynomials in $(\eta, y, \hat{f}) \in \mathbb{R}^{3} \times \mathbb{R}^{3} \times S^{2} \mathbb{C}^{3}$ homogeneous in $\eta$ and linear in $\hat{f}$. For a unit vector $\eta$, the functions $a(\eta, y, \hat{f})$ and $b(\eta, y, \hat{f})$ coincide with left-hand sides of equations (4.13) and (4.14) respectively. Let us now consider the system of equations

$$
a\left(\eta_{i}, y, \hat{f}\right)=0 \quad b\left(\eta_{i}, y, \hat{f}\right)=0, \quad(1 \leq i \leq 3)
$$

for a general point $\left(\eta_{1}, \eta_{2}, \eta_{3} ; y\right) \in \mathbb{R}^{12}$. The determinant of the system is a polynomial in $\left(\eta_{1}, \eta_{2}, \eta_{2} ; y\right)$ homogeneous in each $\eta_{i}$. We have proved that the polynomial is not identically equal to zero. Therefore it does not vanish almost everywhere in $\mathbb{R}^{12}$. Because of the homogeneity, the same is true for unit vectors: the system is nondegenerate for almost all $\left(\eta_{1}, \eta_{2}, \eta_{3} ; y\right) \in\left(\mathbb{S}^{2}\right)^{3} \times \mathbb{R}^{3}$.

Let us return to considering system (5.3) for an orthonormal basis $\left(\eta_{1}, \eta_{2}, \eta_{3}\right)$. It gives us a reconstruction algorithm. Indeed, given the data $\left(\left.K f\right|_{L_{\eta_{1}}^{3}},\left.K f\right|_{L_{\eta_{2}}^{3}},\left.K f\right|_{L_{\eta_{3}}^{3}}\right)$ for a trace-free tensor field $f \in$ $\mathcal{S}\left(\mathbb{R}^{3} ; S^{2} \mathbb{C}^{3}\right)$, right-hand sides $\lambda_{i}(y)$ and $\mu_{i}(y)$ of equations (5.3) are effectively recovered by formulas (4.15)-(4.18). Let us arrange components of $\hat{f}$ to the column-vector $\hat{f}=\left(\hat{f}_{11}, \hat{f}_{12}, \hat{f}_{13}, \hat{f}_{22}, \hat{f}_{23}, \hat{f}_{33}\right)^{t}$. Then the solution to system (5.3) can be represented analytically in the form

$$
\hat{f}(y)=(\operatorname{det}(y))^{-1} M(y)(\lambda(y), \mu(y))^{t}
$$

with some $6 \times 6$-matrix $M(y)$ polynomially depending on $y$, where $\operatorname{det}(y)$ is the determinant of the system. This gives us $\left.\hat{f}\right|_{\mathbb{R}^{3} \backslash \Gamma}$, where

$$
\Gamma=\left\{y \in \mathbb{R}^{3} \mid \operatorname{det}(y)=0\right\}=\left\{y \in \mathbb{R}^{3} \mid y_{1} y_{2} y_{3}=0\right\} .
$$

The data $\left.\hat{f}\right|_{\mathbb{R}^{3} \backslash \Gamma}$ are sufficient for recovering $f$ by applying the inverse Fourier transform.

We summarize this result in the following theorem

TheOREM 5.1. A trace-free tensor field $f \in \mathcal{S}\left(\mathbb{R}^{3} ; S^{2} \mathbb{C}^{3}\right)$ is uniquely determined by the data $\left(\left.K f\right|_{L_{\eta_{1}}^{3}},\left.K f\right|_{L_{\eta_{2}}^{3}},\left.K f\right|_{L_{\eta_{3}}^{3}}\right)$ for a generic choice of three vectors $\left(\eta_{1}, \eta_{2}, \eta_{3}\right)$, in particular an orthogonal basis is sufficient.

Of course, this reconstruction algorithm possesses the following instability: in the presence of measurement errors in the data, the accuracy in recovering $\hat{f}(y)$ decreases as the point $y$ approaches the surface $\Gamma$.

\section{System for six directions}

Here, we investigate the system obtained by combining equations (4.13)-(4.14) for six unit vectors of family (2.6). We use again the abbreviated notations $\lambda_{i}=\lambda_{\eta_{i}}$ and $\mu_{i}=\mu_{\eta_{i}}$.

We start with the following observation: six equations (4.14) give a possibility for the stable reconstruction of mixed components $\hat{f}_{j k}(j \neq k)$. 
For $\eta=\eta_{3}=\frac{1}{\sqrt{2}}(1,1,0)$, we have $\pi_{\eta} y=y-\langle\eta, y\rangle \eta=\frac{1}{2}\left(y_{1}-y_{2}, y_{2}-y_{1}, 2 y_{3}\right)$ and

$$
\begin{aligned}
&\left|\pi_{\eta} y\right|^{2}\langle\hat{f} \eta, \eta\rangle-\left\langle\hat{f} \pi_{\eta} y, \pi_{\eta} y\right\rangle=\frac{1}{4}\left(\left(y_{1}-y_{2}\right)^{2}+2 y_{3}^{2}\right)\left(\hat{f}_{11}+2 \hat{f}_{12}+\hat{f}_{22}\right)-\frac{1}{4}\left(\left(y_{1}-y_{2}\right)^{2}\left(\hat{f}_{11}+\hat{f}_{22}\right)\right. \\
&\left.+4 y_{3}^{2} \hat{f}_{33}-2\left(y_{1}-y_{2}\right)^{2} \hat{f}_{12}+4\left(y_{1}-y_{2}\right) y_{3} \hat{f}_{13}-4\left(y_{1}-y_{2}\right) y_{3} \hat{f}_{23}\right) \\
&=\frac{1}{2} y_{3}^{2}\left(\hat{f}_{11}+\hat{f}_{22}\right)-y_{3}^{2} \hat{f}_{33}+\left(\left(y_{1}-y_{2}\right)^{2}+y_{3}^{2}\right) \hat{f}_{12}-\left(y_{1}-y_{2}\right) y_{3} \hat{f}_{13}+\left(y_{1}-y_{2}\right) y_{3} \hat{f}_{23} .
\end{aligned}
$$

Using the relation $\operatorname{tr} \hat{f}=0$, this can be written as

$$
\left|\pi_{\eta} y\right|^{2}\langle\hat{f} \eta, \eta\rangle-\left\langle\hat{f} \pi_{\eta} y, \pi_{\eta} y\right\rangle=\left(\left(y_{1}-y_{2}\right)^{2}+y_{3}^{2}\right) \hat{f}_{12}-\left(y_{1}-y_{2}\right) y_{3} \hat{f}_{13}+\left(y_{1}-y_{2}\right) y_{3} \hat{f}_{23}-\frac{3}{2} y_{3}^{2} \hat{f}_{33} .
$$

Therefore equation (4.14) for $\eta_{3}$ is as follows:

$$
\left(\left(y_{1}-y_{2}\right)^{2}+y_{3}^{2}\right) \hat{f}_{12}-\left(y_{1}-y_{2}\right) y_{3} \hat{f}_{13}+\left(y_{1}-y_{2}\right) y_{3} \hat{f}_{23}-\frac{3}{2} y_{3}^{2} \hat{f}_{33}=\mu_{3} .
$$

The corresponding equation for $\eta_{6}=\frac{1}{\sqrt{2}}(1,-1,0)$ is derived in the same way

$$
\left(\left(y_{1}+y_{2}\right)^{2}+y_{3}^{2}\right) \hat{f}_{12}+\left(y_{1}+y_{2}\right) y_{3} \hat{f}_{13}+\left(y_{1}+y_{2}\right) y_{3} \hat{f}_{23}+\frac{3}{2} y_{3}^{2} \hat{f}_{33}=-\mu_{6} .
$$

Eliminating $\hat{f}_{33}$ from last two equations, we obtain the equation containing only mixed components

$$
|y|^{2} \hat{f}_{12}+y_{2} y_{3} \hat{f}_{13}+y_{1} y_{3} \hat{f}_{23}=\left(\mu_{3}-\mu_{6}\right) / 2 .
$$

By cyclic transposing indices on the left-hand side, we obtain two similar equations for pairs $\left(\eta_{2}, \eta_{5}\right)$ and $\left(\eta_{1}, \eta_{4}\right)$ of family $(2.6)$. In such the way we arrive to the system

$$
\left\{\begin{array}{l}
|y|^{2} \hat{f}_{12}+y_{2} y_{3} \hat{f}_{13}+y_{1} y_{3} \hat{f}_{23}=\left(\mu_{3}-\mu_{6}\right) / 2 \\
y_{2} y_{3} \hat{f}_{12}+|y|^{2} \hat{f}_{13}+y_{1} y_{2} \hat{f}_{23}=\left(\mu_{2}-\mu_{5}\right) / 2 \\
y_{1} y_{3} \hat{f}_{12}+y_{1} y_{2} \hat{f}_{13}+|y|^{2} \hat{f}_{23}=\left(\mu_{1}-\mu_{4}\right) / 2
\end{array}\right.
$$

System (6.1) is nondegenerate at every point $0 \neq y \in \mathbb{R}^{3}$. Indeed, the determinant of the system admits the estimate det $\geq 2|y|^{6} / 3$. Therefore system (6.1) is uniquely solvable for any $y \neq 0$ and the solution satisfies the stability estimate

$$
\left|\hat{f}_{j k}(y)\right| \leq \frac{3}{2}|y|^{-2} \sum_{i=1}^{3}\left|\mu_{i}(y)-\mu_{i+3}(y)\right| \quad(j \neq k) .
$$

Before going further, let us give a remark on the singularity of system (6.1) at the point $y=0$. Such singularities are unavoidable in our approach. Indeed, all coefficients of our main equations (4.13)-(4.14) vanish at $y=0$. This phenomenon is actually caused by the following fact: the tangential part of a vector (tensor) field has a singularity at the origin; see Sections 2.2 and 3.2 of [12] for details. Therefore let us agree to disregard singularities at the origin and consider systems with such singularities as nondegenerate systems.

Assuming mixed components $\hat{f}_{j k}(j \neq k)$ have been stably recovered, we use six equations (4.13) for a stable reconstruction of the diagonal components $\hat{f}_{j j}$.

Equations (4.13) for $\eta_{3}$ and $\eta_{6}$ can be easily written down:

$$
\begin{aligned}
& \left(y_{1}-y_{2}\right)\left(\hat{f}_{11}-\hat{f}_{22}\right)+2 y_{3}\left(\hat{f}_{13}+\hat{f}_{23}\right)=2 \sqrt{2} \lambda_{3}, \\
& \left(y_{1}+y_{2}\right)\left(\hat{f}_{11}-\hat{f}_{22}\right)+2 y_{3}\left(\hat{f}_{13}-\hat{f}_{23}\right)=2 \sqrt{2} \lambda_{6} .
\end{aligned}
$$


This system is equivalent to the following one:

$$
\begin{aligned}
& y_{1}\left(\hat{f}_{11}-\hat{f}_{22}\right)=\sqrt{2}\left(\lambda_{3}+\lambda_{6}\right)-2 y_{3} \hat{f}_{13}, \\
& y_{2}\left(\hat{f}_{11}-\hat{f}_{22}\right)=\sqrt{2}\left(\lambda_{6}-\lambda_{3}\right)+2 y_{3} \hat{f}_{23} .
\end{aligned}
$$

By cyclic transposing indices, we write similar equations for pairs $\left(\eta_{2}, \eta_{5}\right)$ and $\left(\eta_{1}, \eta_{4}\right)$. In such the way we obtain the system

$$
\left\{\begin{array}{l}
y_{1}\left(\hat{f}_{11}-\hat{f}_{22}\right)=\sqrt{2}\left(\lambda_{3}+\lambda_{6}\right)-2 y_{3} \hat{f}_{13}, \\
y_{2}\left(\hat{f}_{11}-\hat{f}_{22}\right)=\sqrt{2}\left(\lambda_{6}-\lambda_{3}\right)+2 y_{3} \hat{f}_{23}, \\
y_{3}\left(\hat{f}_{33}-\hat{f}_{11}\right)=\sqrt{2}\left(\lambda_{2}+\lambda_{5}\right)-2 y_{2} \hat{f}_{23}, \\
y_{1}\left(\hat{f}_{33}-\hat{f}_{11}\right)=\sqrt{2}\left(\lambda_{5}-\lambda_{2}\right)+2 y_{2} \hat{f}_{12}, \\
y_{2}\left(\hat{f}_{22}-\hat{f}_{33}\right)=\sqrt{2}\left(\lambda_{1}+\lambda_{4}\right)-2 y_{1} \hat{f}_{12}, \\
y_{3}\left(\hat{f}_{22}-\hat{f}_{33}\right)=\sqrt{2}\left(\lambda_{4}-\lambda_{1}\right)+2 y_{1} \hat{f}_{13} .
\end{array}\right.
$$

Take the sum of third and sixth equations of (6.3)

$$
y_{3}\left(\hat{f}_{11}-\hat{f}_{22}\right)=\sqrt{2}\left(\lambda_{1}-\lambda_{2}-\lambda_{4}-\lambda_{5}\right)-2 y_{1} \hat{f}_{13}+2 y_{2} \hat{f}_{23}
$$

and consider the system obtained by uniting this equation with first two equations of (6.3)

$$
\begin{aligned}
& y_{1}\left(\hat{f}_{11}-\hat{f}_{22}\right)=\sqrt{2}\left(\lambda_{3}+\lambda_{6}\right)-2 y_{3} \hat{f}_{13}, \\
& y_{2}\left(\hat{f}_{11}-\hat{f}_{22}\right)=\sqrt{2}\left(\lambda_{6}-\lambda_{3}\right)+2 y_{3} \hat{f}_{23}, \\
& y_{3}\left(\hat{f}_{11}-\hat{f}_{22}\right)=\sqrt{2}\left(\lambda_{1}-\lambda_{2}-\lambda_{4}-\lambda_{5}\right)-2 y_{1} \hat{f}_{13}+2 y_{2} \hat{f}_{23} .
\end{aligned}
$$

Multiplying the first (second, third) of these equations by $y_{1}$ (by $y_{2}, y_{3}$ ) and summing results, we obtain

$$
|y|^{2}\left(\hat{f}_{11}-\hat{f}_{22}\right)=\sqrt{2}\left(\left(y_{1}-y_{2}\right) \lambda_{3}+\left(y_{1}+y_{2}\right) \lambda_{6}+y_{3}\left(\lambda_{1}-\lambda_{2}-\lambda_{4}-\lambda_{5}\right)\right)-4 y_{1} y_{3} \hat{f}_{13}+4 y_{2} y_{3} \hat{f}_{23} .
$$

Quite similarly, the following equation is derived from (6.3):

$$
|y|^{2}\left(\hat{f}_{11}-\hat{f}_{33}\right)=\sqrt{2}\left(\left(y_{1}-y_{3}\right) \lambda_{2}-\left(y_{1}+y_{3}\right) \lambda_{5}-y_{2}\left(\lambda_{3}-\lambda_{1}-\lambda_{4}-\lambda_{6}\right)\right)-4 y_{1} y_{2} \hat{f}_{12}+4 y_{2} y_{3} \hat{f}_{23} .
$$

Taking the sum of last two equations and using the relation $\operatorname{tr} \hat{f}=0$, we obtain the stable reconstruction formula for $\hat{f}_{11}$

$$
\begin{aligned}
\hat{f}_{11} & =\frac{\sqrt{2}}{3|y|^{2}}\left(\left(y_{2}+y_{3}\right) \lambda_{1}+\left(y_{1}-2 y_{3}\right) \lambda_{2}+\left(y_{1}-2 y_{2}\right) \lambda_{3}+\left(y_{2}-y_{3}\right) \lambda_{4}\right. \\
& \left.-\left(y_{1}+2 y_{3}\right) \lambda_{5}+\left(y_{1}+2 y_{2}\right) \lambda_{6}\right)-\frac{4}{3|y|^{2}}\left(y_{1} y_{2} \hat{f}_{12}+y_{1} y_{3} \hat{f}_{13}-2 y_{2} y_{3} \hat{f}_{23}\right) .
\end{aligned}
$$

The corresponding formulas for $\hat{f}_{22}$ and $\hat{f}_{33}$ are obtained in the same way

$$
\begin{aligned}
\hat{f}_{22} & =\frac{\sqrt{2}}{3|y|^{2}}\left(\left(y_{2}-2 y_{3}\right) \lambda_{1}+\left(y_{1}+y_{3}\right) \lambda_{2}-\left(2 y_{1}-y_{2}\right) \lambda_{3}+\left(y_{2}+2 y_{3}\right) \lambda_{4}\right. \\
& \left.-\left(y_{1}-y_{3}\right) \lambda_{5}-\left(2 y_{1}+y_{2}\right) \lambda_{6}\right)-\frac{4}{3|y|^{2}}\left(y_{1} y_{2} \hat{f}_{12}-2 y_{1} y_{3} \hat{f}_{13}+y_{2} y_{3} \hat{f}_{23}\right),
\end{aligned}
$$




$$
\begin{aligned}
\hat{f}_{33} & =\frac{\sqrt{2}}{3|y|^{2}}\left(-\left(2 y_{2}-y_{3}\right) \lambda_{1}-\left(2 y_{1}-y_{3}\right) \lambda_{2}+\left(y_{1}+y_{2}\right) \lambda_{3}-\left(2 y_{2}+y_{3}\right) \lambda_{4}\right. \\
& \left.+\left(2 y_{1}+y_{3}\right) \lambda_{5}+\left(y_{1}-y_{2}\right) \lambda_{6}\right)-\frac{4}{3|y|^{2}}\left(-2 y_{1} y_{2} \hat{f}_{12}+y_{1} y_{3} \hat{f}_{13}+y_{2} y_{3} \hat{f}_{23}\right) .
\end{aligned}
$$

Formulas (6.4)-(6.6) imply the stability estimate

$$
\left|\hat{f}_{11}(y)\right|+\left|\hat{f}_{22}(y)\right|+\left|\hat{f}_{33}(y)\right| \leq C^{\prime}\left(|y|^{-1} \sum_{i=1}^{6}\left|\lambda_{i}(y)\right|+\left|\hat{f}_{12}(y)\right|+\left|\hat{f}_{13}(y)\right|+\left|\hat{f}_{23}(y)\right|\right)
$$

with some universal constant $C^{\prime}$. Together with (6.2), this gives the final stability estimate

$$
|\hat{f}(y)| \leq C\left(|y|^{-1} \sum_{i=1}^{6}\left|\lambda_{i}(y)\right|+|y|^{-2} \sum_{i=1}^{3}\left|\mu_{i}(y)-\mu_{i+3}(y)\right|\right)
$$

with some universal constant $C$.

Similarly we obtain the estimate on the Frobenius norm of $\hat{f}$

$$
|\hat{f}(y)|^{2} \leq C^{\prime \prime}\left(|y|^{-2} \sum_{i=1}^{6}\left|\lambda_{i}(y)\right|^{2}+|y|^{-4} \sum_{i=1}^{3}\left|\mu_{i}(y)-\mu_{i+3}(y)\right|^{2}\right)
$$

which gives stability estimates in $L^{2}$ based Sobolev spaces.

We have now proved the following theorem

THEOREM 6.1. For the family (2.6) of unit vectors the data $\left(\left.K f\right|_{L_{\eta_{1}}^{3}}, \ldots,\left.K f\right|_{L_{\eta_{6}}^{3}}\right)$ uniquely determines a trace-free field $f \in \mathcal{S}\left(\mathbb{R}^{3} ; S^{2} \mathbb{C}^{3}\right)$ with a stable, explicit reconstruction formula. Stability estimates are given by (6.8) and (6.9).

Summary of the algorithm. For the reader's convenience, we summarize here all essential steps needed for the numerical realization of the algorithm implied by Theorem 6.1

(1) Identifying $L_{\eta_{i}}^{3}$ with $\mathbb{R} \times T \mathbb{S}_{\eta_{i}}^{1}$ as at the end of Section 3 and using formulas (4.17)-(4.18), represent the data as the family $\left(K_{\eta_{1}}^{j} f, \ldots, K_{\eta_{6}}^{j} f\right)(j=1,2)$ of functions $K_{\eta_{i}}^{j} f \in \mathcal{S}\left(\mathbb{R} \times T \mathbb{S}_{\eta_{i}}^{1}\right)$. Evaluate the derivatives $\partial\left(K_{\eta_{i}}^{1} f\right) / \partial p$ as explained in Lemma 4.1.

(2) Using formula (4.8), evaluate the functions $\left(B_{\eta_{i}}\left(\partial K_{\eta_{i}}^{1} f / \partial p\right)\right)(x)$ and $B_{\eta_{i}} K_{\eta_{i}}^{2} f(x)(1 \leq i \leq 6)$ by multiple applying the two-dimensional back projection operator $B_{\eta_{i}}$ for every value of $\left\langle x, \eta_{i}\right\rangle$ (practically, for $\left\langle x, \eta_{i}\right\rangle$ belonging to a finite grid).

(3) Evaluate $F\left[B_{\eta_{i}}\left(\partial K_{\eta_{i}}^{1} f / \partial p\right)\right]$ and $F\left[B_{\eta_{i}} K_{\eta_{i}}^{2} f\right](1 \leq i \leq 6)$ by applying the 3D Fourier transform and then calculate the functions $\lambda_{i}=\lambda_{\eta_{i}}$ and $\mu_{i}=\mu_{\eta_{i}}$ by formulas (4.15)-(4.16).

(4) Find mixed components $\hat{f}_{j k}(y)(j \neq k)$ by solving system (6.1) and then find diagonal components $\hat{f}_{j j}$ by formulas $(6.4)-(6.6)$.

(5) Find $f(x)$ by applying the inverse Fourier transform.

\section{Consistency conditions}

Let us now discuss the question on consistency conditions in the case of family (2.6): given a family of twelve functions $\varphi_{i}^{j} \in \mathcal{S}\left(\mathbb{R} \times \mathbb{S}_{\eta_{i}}^{1}\right)(1 \leq i \leq 6,1 \leq j \leq 2)$, does there exist a trace-free tensor field $f \in \mathcal{S}\left(\mathbb{R}^{3} ; \mathbb{C}^{3}\right)$ such that $\varphi_{i}^{j}=K_{\eta_{i}}^{j} f$ ? Actually we discuss consistency conditions in terms of functions $\lambda_{i}=\lambda_{\eta_{i}}$ and $\mu_{i}=\mu_{\eta_{i}}(1 \leq i \leq 6)$ defined by (4.15) and (4.16). There are three kinds of consistency conditions which can be called the local, global, and algebraic conditions respectively.

(1) Local consistency conditions. First of all we observe that only the differences

$$
\tilde{\mu}_{i}=\mu_{i}-\mu_{i+3} \quad(1 \leq i \leq 3)
$$


are used in our algorithm as is seen from (6.1). Also only three linear combinations of $\lambda_{i}$ 's

$$
\begin{aligned}
\tilde{\lambda}_{1}= & \left(y_{2}+y_{3}\right) \lambda_{1}+\left(y_{1}-2 y_{3}\right) \lambda_{2}+\left(y_{1}-2 y_{2}\right) \lambda_{3} \\
& +\left(y_{2}-y_{3}\right) \lambda_{4}-\left(y_{1}+2 y_{3}\right) \lambda_{5}+\left(y_{1}+2 y_{2}\right) \lambda_{6}, \\
\tilde{\lambda}_{2}= & \left(y_{2}-2 y_{3}\right) \lambda_{1}+\left(y_{1}+y_{3}\right) \lambda_{2}-\left(2 y_{1}-y_{2}\right) \lambda_{3} \\
& +\left(y_{2}+2 y_{3}\right) \lambda_{4}-\left(y_{1}-y_{3}\right) \lambda_{5}-\left(2 y_{1}+y_{2}\right) \lambda_{6}, \\
\tilde{\lambda}_{3}= & -\left(2 y_{2}-y_{3}\right) \lambda_{1}-\left(2 y_{1}-y_{3}\right) \lambda_{2}+\left(y_{1}+y_{2}\right) \lambda_{3} \\
& -\left(2 y_{2}+y_{3}\right) \lambda_{4}+\left(2 y_{1}+y_{3}\right) \lambda_{5}+\left(y_{1}-y_{2}\right) \lambda_{6}
\end{aligned}
$$

participate in the algorithm as is seen from $(6.4)-(6.6)$. The family $\left(\tilde{\lambda}_{1}(y), \ldots, \tilde{\mu}_{3}(y)\right)$ must be such that the field $\hat{f}(y)$, obtained by solving system (6.1) and substituting the solution into (6.4)-(6.6), belongs to $\mathcal{S}\left(\mathbb{R}^{3} ; S^{2} \mathbb{C}^{3}\right)$. This requirement can be considered as the first consistency condition. Let us refer to the requirement as "the local consistency condition". It implies (but is not equivalent to) the following fact: the functions $\tilde{\lambda}_{i}$ and $\tilde{\mu}_{i}$ belong to the subspace of $\mathcal{S}\left(\mathbb{R}^{3}\right)$ consisting of functions vanishing at the origin together with first order derivatives. Let us also mention that formulas (4.15)-(4.16) do not guarantee any good behavior of the functions $\lambda_{\eta}(y)$ and $\mu_{\eta}(y)$ near the origin since the Fourier transform of $B_{\eta} K_{\eta}^{j} f$ can be singular at the origin, compare with the remark after formula (4.5).

(2) Global consistency condition. It can be very implicitly described as follows. For a family $\left(\tilde{\lambda}_{1}, \ldots, \tilde{\mu}_{3}\right)$ of six functions satisfying the local consistency conditions, let $\hat{f}$ be the solution to systems (6.1) and (6.4)-(6.6), and let $\left(\lambda_{i}=K_{\eta_{i}}^{1} f, \mu_{i}=K_{\eta_{i}}^{2} f \mid 1 \leq i \leq 6\right)$ be the corresponding data for the field $f=F^{-1}[\hat{f}]$. We have thus defined some linear operator

$$
A:\left(\tilde{\lambda}_{1}, \ldots, \tilde{\mu}_{3}\right) \mapsto\left(\lambda_{1}, \ldots, \mu_{6}\right) .
$$

Define also the operator

$$
B:\left(\lambda_{1}, \ldots, \mu_{6}\right) \mapsto\left(\tilde{\lambda}_{1}, \ldots, \tilde{\mu}_{3}\right)
$$

by formulas (7.1)-(7.2). The global consistency condition can be formulated as follows: our data $\mathcal{D}=\left(\lambda_{1}, \ldots, \mu_{6}\right)$ must satisfy $A B \mathcal{D}=\mathcal{D}$. Our algorithm is independent of this condition since only the data $\left(\tilde{\lambda}_{1}, \ldots, \tilde{\mu}_{3}\right)$ are used in the algorithm.

(3) Algebraic consistency conditions. First of all we observe that the equation $\operatorname{tr} \hat{f}=0$ is satisfied by expressions (6.4)-(6.6) as can be checked by substitution. We have the system of 12 linear algebraic equations (4.13)-(4.14) in six unknowns $\hat{f}_{j k}(y)$. Therefore right-hand sides $\left(\lambda_{1}, \ldots, \lambda_{6}, \mu_{1}, \ldots, \mu_{6}\right)$ must satisfy 6 linear algebraic equations (with coefficients depending on $y$ ). By substitute expressions (6.4)-(6.6) into equations (6.3) and eliminating each $\hat{f}_{i i}$ from a pair of equations we obtain consistency conditions on the $\lambda_{i}$. Although there are three equations (6.3) this only results in one independent consistency condition

$$
\begin{aligned}
& y_{2} y_{3}\left(-y_{2}\left(y_{1}^{2}-y_{2}^{2}+y_{3}^{2}\right)+y_{3}\left(y_{1}^{2}+y_{2}^{2}-y_{3}^{2}\right)\right) \lambda_{1} \\
+ & y_{1} y_{3}\left(-y_{3}\left(y_{1}^{2}+y_{2}^{2}-y_{3}^{2}\right)+y_{1}\left(-y_{1}^{2}+y_{2}^{2}+y_{3}^{2}\right)\right) \lambda_{2} \\
+ & y_{1} y_{2}\left(-y_{1}\left(-y_{1}^{2}+y_{2}^{2}+y_{3}^{2}\right)+y_{2}\left(y_{1}^{2}-y_{2}^{2}+y_{3}^{2}\right)\right) \lambda_{3} \\
+ & y_{2} y_{3}\left(y_{2}\left(y_{1}^{2}-y_{2}^{2}+y_{3}^{2}\right)+y_{3}\left(y_{1}^{2}+y_{2}^{2}-y_{3}^{2}\right)\right) \lambda_{4} \\
+ & y_{1} y_{3}\left(y_{3}\left(y_{1}^{2}+y_{2}^{2}-y_{3}^{2}\right)+y_{1}\left(-y_{1}^{2}+y_{2}^{2}+y_{3}^{2}\right)\right) \lambda_{5} \\
+ & y_{1} y_{2}\left(y_{1}\left(-y_{1}^{2}+y_{2}^{2}+y_{3}^{2}\right)+y_{2}\left(y_{1}^{2}-y_{2}^{2}+y_{3}^{2}\right)\right) \lambda_{6}=0 .
\end{aligned}
$$


It is invariant under the permutation of indices

$$
1 \mapsto 3 \mapsto 2 \mapsto 1, \quad 4 \mapsto 6 \mapsto 5 \mapsto 4 .
$$

The proof is given in the appendix.

The other algebraic consistency conditions relate both the $\mu_{i}$ and $\lambda_{i}$ and are considerably more complicated.

\section{Conclusions}

We have shown that using the data for the truncated transverse ray transform only for rays orthogonal to six directions it is possible to reconstruct a trace-free tensor field in the Schwarz class. Both the TTRT and the lateral Ray transforms we have used are easily extended from the Schwartz class to the space $\mathcal{E}^{\prime}\left(\mathbb{R}^{3} ; S^{2} \mathbb{C}^{3}\right)$ of compactly supported tensor field-distributions by application of the formal adjoint operators to test functions, following the procedure in [11, Sec 2.14]. Our estimate (6.9) then implies that the inversion procedure is bounded on the Sobolev scale in a way analogous to the scalar X-ray transform [7]. Clearly this implies that some regularization is required for a practical numerical procedure, again taking our queue from the scalar case this would typically be done by a modification of the inversion operator for large $|y|$, although [14] suggests a more systematic approach to regularization for photoelastic applications. The stability of the inversion on Sobolev scales implies in particular that in the important case of tensor fields that are the product of a smooth field with the characteristic function of a bounded Lipschitz domain, a case typical in photoelasticity. Although the solenoidal condition might hold in the interior this allows for the possibility of forces on the boundary of the domain including external loads. Hence the technique could be used in photoelastic tomography provided the characteristic parameter data can be collected with sufficient precision and an apparatus developed that is capable of rotating the specimen a half revolution about six axes of rotation without any of the necessary rays being obscured.

While the method implemented by [2] requires the use of only one dimensional convolution filters followed by a two dimensional back projection on each slice, our method performs a two dimensional back projection on each slice followed by a three dimensional filter coupling the components of the tensor field. In this sense it is more costly, although typically in three dimensional scalar computerized tomography it is the back projection rather than the filter operations that are more time consuming. Our algorithm does however have the advantage that once the back projection operations have been performed the characteristic parameter data are no longer required to be retained in memory. We anticipated that steps (3) and (5) in our algorithm can be implemented efficiently on a rectangular voxel grid using a three dimensional Fast Fourier Transform.

\section{Appendix}

Proof of (7.3). Substituting expressions (6.4)-(6.6) into first two equations of system (6.3), we obtain the equations

$$
\left\{\begin{array}{l}
\left(2 y_{1}^{2}-|y|^{2}\right) \hat{f}_{13}-2 y_{1} y_{2} \hat{f}_{23}=b_{1}, \\
2 y_{1} y_{2} \hat{f}_{13}-\left(2 y_{2}^{2}-|y|^{2}\right) \hat{f}_{23}=b_{2}
\end{array}\right.
$$

with the right-hand sides

$$
\begin{aligned}
& b_{1}=\frac{1}{\sqrt{2}}\left(y_{1}\left(\lambda_{1}-\lambda_{2}-\lambda_{4}-\lambda_{5}\right)+\frac{1}{y_{3}}\left(y_{1}^{2}-y_{1} y_{2}-|y|^{2}\right) \lambda_{3}+\frac{1}{y_{3}}\left(y_{1}^{2}+y_{1} y_{2}-|y|^{2}\right) \lambda_{6}\right), \\
& b_{2}=\frac{1}{\sqrt{2}}\left(y_{2}\left(\lambda_{1}-\lambda_{2}-\lambda_{4}-\lambda_{5}\right)+\frac{1}{y_{3}}\left(y_{1} y_{2}-y_{2}^{2}+|y|^{2}\right) \lambda_{3}+\frac{1}{y_{3}}\left(y_{1} y_{2}+y_{2}^{2}-|y|^{2}\right) \lambda_{6}\right) .
\end{aligned}
$$

We consider (9.1) as a system in unknowns $\hat{f}_{13}$ and $\hat{f}_{23}$. The determinant of the system is

$$
\Delta=\left|\begin{array}{cc}
2 y_{1}^{2}-|y|^{2} & -2 y_{1} y_{2} \\
2 y_{1} y_{2} & -\left(2 y_{2}^{2}-|y|^{2}\right)
\end{array}\right|=|y|^{2}\left(y_{1}^{2}+y_{2}^{2}-y_{3}^{2}\right)
$$


Solve the system by Cramer

$$
\hat{f}_{13}=\frac{1}{\Delta}\left|\begin{array}{cc}
b_{1} & -2 y_{1} y_{2} \\
b_{2} & |y|^{2}-2 y_{2}^{2}
\end{array}\right|=\frac{1}{\Delta}\left(\left(y_{1}^{2}-y_{2}^{2}+y_{3}^{2}\right) b_{1}+2 y_{1} y_{2} b_{2}\right)
$$

Substitute the values of $b_{1}, b_{2}$ and $\Delta$ to obtain

$$
\begin{array}{r}
\hat{f}_{13}=\frac{1}{\sqrt{2}|y|^{2}\left(y_{1}^{2}+y_{2}^{2}-y_{3}^{2}\right)}\left(|y|^{2} y_{1}\left(\lambda_{1}-\lambda_{2}-\lambda_{4}-\lambda_{5}\right)\right. \\
+\frac{1}{y_{3}}\left(y_{1}^{3} y_{2}+y_{1}^{2} y_{2}^{2}-y_{1}^{2} y_{3}^{2}+y_{1} y_{2}^{3}+y_{1} y_{2} y_{3}^{2}+y_{2}^{4}-y_{3}^{4}\right) \lambda_{3} \\
\left.+\frac{1}{y_{3}}\left(-y_{1}^{3} y_{2}+y_{1}^{2} y_{2}^{2}-y_{1}^{2} y_{3}^{2}-y_{1} y_{2}^{3}-y_{1} y_{2} y_{3}^{2}+y_{2}^{4}-y_{3}^{4}\right) \lambda_{6}\right) .
\end{array}
$$

Quite similarly

$$
\begin{aligned}
\hat{f}_{23}=\frac{1}{\sqrt{2}|y|^{2}\left(y_{1}^{2}+y_{2}^{2}-y_{3}^{2}\right)} & \left(-|y|^{2} y_{2}\left(\lambda_{1}-\lambda_{2}-\lambda_{4}-\lambda_{5}\right)\right. \\
+ & \frac{1}{y_{3}}\left(y_{1}^{4}+y_{1}^{3} y_{2}+y_{1}^{2} y_{2}^{2}+y_{1} y_{2}^{3}+y_{1} y_{2} y_{3}^{2}-y_{2}^{2} y_{3}^{2}-y_{3}^{4}\right) \lambda_{3} \\
& \left.+\frac{1}{y_{3}}\left(-y_{1}^{4}+y_{1}^{3} y_{2}-y_{1}^{2} y_{2}^{2}+y_{1} y_{2}^{3}+y_{1} y_{2} y_{3}^{2}+y_{2}^{2} y_{3}^{2}+y_{3}^{4}\right) \lambda_{6}\right) .
\end{aligned}
$$

We do not need to repeat our calculations for third and fourth equations of system (6.3) since they are obtained by applying permutation (7.4) to the first two equations of the system. We just apply permutation (7.4) to formula (9.3) to obtain

$$
\begin{aligned}
\hat{f}_{23}=\frac{1}{\sqrt{2}|y|^{2}\left(y_{1}^{2}-y_{2}^{2}+y_{3}^{2}\right)}\left(|y|^{2} y_{3}\left(-\lambda_{1}+\lambda_{3}-\lambda_{4}-\lambda_{6}\right)\right. \\
+\frac{1}{y_{2}}\left(y_{1}^{4}+y_{1}^{3} y_{3}+y_{1}^{2} y_{3}^{2}+y_{1} y_{2}^{2} y_{3}+y_{1} y_{3}^{3}-y_{2}^{4}-y_{2}^{2} y_{3}^{2}\right) \lambda_{2} \\
\left.+\frac{1}{y_{2}}\left(y_{1}^{4}-y_{1}^{3} y_{3}+y_{1}^{2} y_{3}^{2}-y_{1} y_{2}^{2} y_{3}-y_{1} y_{3}^{3}-y_{2}^{4}-y_{2}^{2} y_{3}^{2}\right) \lambda_{5}\right) .
\end{aligned}
$$

We have thus obtained two different formulas (9.4) and (9.5) for the same $\hat{f}_{23}$. We equate right-hand sides of these formulas

$$
\begin{aligned}
\frac{1}{\sqrt{2}|y|^{2}\left(y_{1}^{2}+y_{2}^{2}-y_{3}^{2}\right)}( & -|y|^{2} y_{2}\left(\lambda_{1}-\lambda_{2}-\lambda_{4}-\lambda_{5}\right) \\
& +\frac{1}{y_{3}}\left(y_{1}^{4}+y_{1}^{3} y_{2}+y_{1}^{2} y_{2}^{2}+y_{1} y_{2}^{3}+y_{1} y_{2} y_{3}^{2}-y_{2}^{2} y_{3}^{2}-y_{3}^{4}\right) \lambda_{3} \\
& \left.+\frac{1}{y_{3}}\left(-y_{1}^{4}+y_{1}^{3} y_{2}-y_{1}^{2} y_{2}^{2}+y_{1} y_{2}^{3}+y_{1} y_{2} y_{3}^{2}+y_{2}^{2} y_{3}^{2}+y_{3}^{4}\right) \lambda_{6}\right) \\
=\frac{1}{\sqrt{2}|y|^{2}\left(y_{1}^{2}-y_{2}^{2}+y_{3}^{2}\right)}\left(|y|^{2} y_{3}\left(-\lambda_{1}+\lambda_{3}-\lambda_{4}-\lambda_{6}\right)\right. & \\
& +\frac{1}{y_{2}}\left(y_{1}^{4}+y_{1}^{3} y_{3}+y_{1}^{2} y_{3}^{2}+y_{1} y_{2}^{2} y_{3}+y_{1} y_{3}^{3}-y_{2}^{4}-y_{2}^{2} y_{3}^{2}\right) \lambda_{2} \\
& \left.+\frac{1}{y_{2}}\left(y_{1}^{4}-y_{1}^{3} y_{3}+y_{1}^{2} y_{3}^{2}-y_{1} y_{2}^{2} y_{3}-y_{1} y_{3}^{3}-y_{2}^{4}-y_{2}^{2} y_{3}^{2}\right) \lambda_{5}\right) .
\end{aligned}
$$


Multiply this equation by the common denominator

$$
\begin{aligned}
\left(y_{1}^{2}-y_{2}^{2}+y_{3}^{2}\right)( & -|y|^{2} y_{2}^{2} y_{3}\left(\lambda_{1}-\lambda_{2}-\lambda_{4}-\lambda_{5}\right) \\
& +y_{2}\left(y_{1}^{4}+y_{1}^{3} y_{2}+y_{1}^{2} y_{2}^{2}+y_{1} y_{2}^{3}+y_{1} y_{2} y_{3}^{2}-y_{2}^{2} y_{3}^{2}-y_{3}^{4}\right) \lambda_{3} \\
& \left.+y_{2}\left(-y_{1}^{4}+y_{1}^{3} y_{2}-y_{1}^{2} y_{2}^{2}+y_{1} y_{2}^{3}+y_{1} y_{2} y_{3}^{2}+y_{2}^{2} y_{3}^{2}+y_{3}^{4}\right) \lambda_{6}\right) \\
=\left(y_{1}^{2}+y_{2}^{2}-y_{3}^{2}\right)( & |y|^{2} y_{2} y_{3}^{2}\left(-\lambda_{1}+\lambda_{3}-\lambda_{4}-\lambda_{6}\right) \\
& +y_{3}\left(y_{1}^{4}+y_{1}^{3} y_{3}+y_{1}^{2} y_{3}^{2}+y_{1} y_{2}^{2} y_{3}+y_{1} y_{3}^{3}-y_{2}^{4}-y_{2}^{2} y_{3}^{2}\right) \lambda_{2} \\
& \left.+y_{3}\left(y_{1}^{4}-y_{1}^{3} y_{3}+y_{1}^{2} y_{3}^{2}-y_{1} y_{2}^{2} y_{3}-y_{1} y_{3}^{3}-y_{2}^{4}-y_{2}^{2} y_{3}^{2}\right) \lambda_{5}\right) .
\end{aligned}
$$

Grouping similar terms, we write this in the form

$$
a_{1} \lambda_{1}+a_{2} \lambda_{2}+a_{3} \lambda_{3}+a_{4} \lambda_{4}+a_{5} \lambda_{5}+a_{6} \lambda_{6}=0,
$$

where

$$
\begin{aligned}
& a_{1}=|y|^{2} y_{2} y_{3}\left(-y_{2}\left(y_{1}^{2}-y_{2}^{2}+y_{3}^{2}\right)+y_{3}\left(y_{1}^{2}+y_{2}^{2}-y_{3}^{2}\right)\right), \\
& a_{2}=y_{3}\left(|y|^{2} y_{2}^{2}\left(y_{1}^{2}-y_{2}^{2}+y_{3}^{2}\right)\right. \\
& \left.-\left(y_{1}^{2}+y_{2}^{2}-y_{3}^{2}\right)\left(y_{1}^{4}+y_{1}^{3} y_{3}+y_{1}^{2} y_{3}^{2}+y_{1} y_{2}^{2} y_{3}+y_{1} y_{3}^{3}-y_{2}^{4}-y_{2}^{2} y_{3}^{2}\right)\right), \\
& a_{3}=y_{2}\left(\left(y_{1}^{2}-y_{2}^{2}+y_{3}^{2}\right)\left(y_{1}^{4}+y_{1}^{3} y_{2}+y_{1}^{2} y_{2}^{2}+y_{1} y_{2}^{3}+y_{1} y_{2} y_{3}^{2}-y_{2}^{2} y_{3}^{2}-y_{3}^{4}\right)\right. \\
& \left.-|y|^{2} y_{3}^{2}\left(y_{1}^{2}+y_{2}^{2}-y_{3}^{2}\right)\right) \text {, } \\
& a_{4}=|y|^{2} y_{2} y_{3}\left(y_{2}\left(y_{1}^{2}-y_{2}^{2}+y_{3}^{2}\right)+y_{3}\left(y_{1}^{2}+y_{2}^{2}-y_{3}^{2}\right)\right) \text {, } \\
& a_{5}=y_{3}\left(|y|^{2} y_{2}^{2}\left(y_{1}^{2}-y_{2}^{2}+y_{3}^{2}\right)\right. \\
& \left.-\left(y_{1}^{2}+y_{2}^{2}-y_{3}^{2}\right)\left(y_{1}^{4}-y_{1}^{3} y_{3}+y_{1}^{2} y_{3}^{2}-y_{1} y_{2}^{2} y_{3}-y_{1} y_{3}^{3}-y_{2}^{4}-y_{2}^{2} y_{3}^{2}\right)\right), \\
& a_{6}=y_{2}\left(\left(y_{1}^{2}-y_{2}^{2}+y_{3}^{2}\right)\left(-y_{1}^{4}+y_{1}^{3} y_{2}-y_{1}^{2} y_{2}^{2}+y_{1} y_{2}^{3}+y_{1} y_{2} y_{3}^{2}+y_{2}^{2} y_{3}^{2}+y_{3}^{4}\right)\right. \\
& \left.+|y|^{2} y_{3}^{2}\left(y_{1}^{2}+y_{2}^{2}-y_{3}^{2}\right)\right) \text {. }
\end{aligned}
$$

By straightforward but cumbersome calculations, formulas (9.7) are transformed to the form

$$
\begin{aligned}
& a_{1}=|y|^{2} y_{2} y_{3}\left(-y_{2}\left(y_{1}^{2}-y_{2}^{2}+y_{3}^{2}\right)+y_{3}\left(y_{1}^{2}+y_{2}^{2}-y_{3}^{2}\right)\right), \\
& a_{2}=|y|^{2} y_{1} y_{3}\left(-y_{3}\left(y_{1}^{2}+y_{2}^{2}-y_{3}^{2}\right)+y_{1}\left(-y_{1}^{2}+y_{2}^{2}+y_{3}^{2}\right)\right), \\
& a_{3}=|y|^{2} y_{1} y_{2}\left(-y_{1}\left(-y_{1}^{2}+y_{2}^{2}+y_{3}^{2}\right)+y_{2}\left(y_{1}^{2}-y_{2}^{2}+y_{3}^{2}\right)\right), \\
& a_{4}=|y|^{2} y_{2} y_{3}\left(y_{2}\left(y_{1}^{2}-y_{2}^{2}+y_{3}^{2}\right)+y_{3}\left(y_{1}^{2}+y_{2}^{2}-y_{3}^{2}\right)\right), \\
& a_{5}=|y|^{2} y_{1} y_{3}\left(y_{3}\left(y_{1}^{2}+y_{2}^{2}-y_{3}^{2}\right)+y_{1}\left(-y_{1}^{2}+y_{2}^{2}+y_{3}^{2}\right)\right), \\
& a_{6}=|y|^{2} y_{1} y_{2}\left(y_{1}\left(-y_{1}^{2}+y_{2}^{2}+y_{3}^{2}\right)+y_{2}\left(y_{1}^{2}-y_{2}^{2}+y_{3}^{2}\right)\right) .
\end{aligned}
$$

Substituting values (9.8) into (9.6), we arrive at (7.3).

\section{References}

[1] H Aben, Integrated Photoelasticity, McGraw-Hill, New York, 1979.

[2] HK Aben, Kerr effect tomography for general axisymmetric field, Appl. Opt., 26, 2921-2924, 1987. 
[3] H Aben, A Errapart, L Ainola, and J Anton, Photoelastic tomography for residual stress measurement in glass, Opt. Eng., 44, 093601, 2005.

[4] H Fessler, An assessment of frozen stress photoelasticity, J. Strain Analysis, 27 (3), 123-126, 1993.

[5] H Hammer, Characteristic parameters in integrated photoelasticity: an application of Poincaré's equivalence theorem, J. Mod. Opt., 51, 597-618, 2004.

[6] WRB Lionheart and CJP Newton, Analysis of the inverse problem for determining nematic liquid crystal director profiles from optical measurements using singular value decomposition, New J. Phys. 9, 63, 2007.

[7] F Natterer, The Mathematics of Computerized Tomography, SIAM, Philadelphia PA, 2001.

[8] R Novikov, and V Sharafutdinov, On the problem of polarization tomography: I, Inverse Problems, 23, 1229$1257,2007$.

[9] VV Pikalov and TS Melnikova, Tomography of Plasma, Nauka SO, Novosibirsk, 1995.

[10] VA Sharafutdinov, The method of integral photo-elasticity in the case of weak optical anisotropy. Eesti NSV Tead. Akad. Toimetised Füüs.-Mat., 38 (4), 379-389, 468, 1989 (Russian, English and Estonian summaries).

[11] V Sharafutdinov, Integral Geometry of Tensor Fields VSP, Utrecht, the Netherlands, 1994.

[12] V Sharafutdinov, Slice-by-slice reconstruction algorithm for vector tomography with incomplete data, Inverse Problems, 23, 2603-2627, 2007.

[13] VA Sharafutdinov, The problem of polarization tomography: II, Inverse Problems, 24, 035010, 2008.

[14] D Szotten, WRB Lionheart and RA Tomlinson, Tomographic reconstruction of stress from photoelastic measurements using elastic regularization, MIMS e-print 2006.5, 2006.

[15] RA Tomlinson, EA Patterson, The Use of Phase-Stepping for the Measurement of Characteristic Parameters in Integrated Photoelasticity, Experimental Mechanics, 42 (1), 43-50, 2002.

School of Mathematics, University of Manchester, Manchester M13 9PL

E-mail address: bill.lionheart@manchester.ac.uk

Sobolev Institute of Mathematics, 4 Koptjug Avenue, Novosibirsk, 630090, Russia

E-mail address: sharaf@math.nsc.ru 